\title{
A large-scale multiomics analysis of wheat stem solidness and the wheat stem sawfly feeding response, and syntenic associations in barley, Brachypodium, and rice
}

\author{
Sezgi Biyiklioglu ${ }^{1} \cdot$ Burcu Alptekin ${ }^{1} \cdot$ B. Ani Akpinar ${ }^{1} \cdot$ Andrea C. Varella $^{2} \cdot$ Megan L. Hofland $^{3} \cdot$ David K. Weaver $^{3} \cdot$ \\ Brian Bothner ${ }^{4} \cdot$ Hikmet Budak $^{1}$ (D)
}

Received: 14 August 2017 / Revised: 13 December 2017 / Accepted: 14 December 2017 / Published online: 22 February 2018

(C) The Author(s) 2018. This article is an open access publication

\begin{abstract}
The wheat stem sawfly (WSS), Cephus cinctus Norton (Hymenoptera: Cephidae), is an important pest of wheat and other cereals, threatening the quality and quantity of grain production. WSS larvae feed and develop inside the stem where they are protected from the external environment; therefore, pest management strategies primarily rely on host plant resistance. A major locus on the long arm of wheat chromosome 3B underlies most of the variation in stem solidness; however, the impact of stem solidness on WSS feeding has not been completely characterized. Here, we used a multiomics approach to examine the response to WSS in both solid- and semi-solid-stemmed wheat varieties. The combined transcriptomic, proteomic, and metabolomic data revealed that two important molecular pathways, phenylpropanoid and phosphate pentose, are involved in plant defense against WSS. We also detected a general downregulation of several key defense transcripts, including those encoding secondary metabolites such as DIMBOA, tricetin, and lignin, which suggested that the WSS larva might interfere with plant defense. We comparatively analyzed the stem solidness genomic region known to be associated with WSS tolerance in wild emmer, durum, and bread wheats, and described syntenic regions in the close relatives barley, Brachypodium, and rice. Additionally, microRNAs identified from the same genomic region revealed potential regulatory pathways associated with the WSS response. We propose a model outlining the molecular responses of the WSS-wheat interactions. These findings provide insight into the link between stem solidness and WSS feeding at the molecular level.
\end{abstract}

Keywords Brachypodium · Hordeum vulgare · Insectresistance · Oryza sativa $\cdot$ Stem solidness $\cdot$ SSt1 $\cdot$ Triticum aestivum $\cdot$ Wheat stem sawfly feeding

Electronic supplementary material The online version of this article (https://doi.org/10.1007/s10142-017-0585-5) contains supplementary material, which is available to authorized users.

Hikmet Budak

hikmet.budak@montana.edu

1 Cereal Genomics Laboratory, Department of Plant Sciences and Plant Pathology, Montana State University, Bozeman, MT, USA

2 Department of Plant Sciences and Plant Pathology, Montana State University, Bozeman, MT, USA

3 Wheat Stem Sawfly Laboratory, Department of Land Resources and Environmental Sciences, Montana State University, Bozeman, MT, USA

4 Department of Chemistry and Biochemistry, Montana State University, Bozeman, MT, USA

\section{Introduction}

Wheat (Triticum aestivum) is a staple food for $30 \%$ of the global population and is the most extensively grown crop in the world, ranking third in terms of production with an annual output of over 700 million tons (http://faostat3.fao.org/). Biotic stresses are responsible for up to $20 \%$ of the yield loss in wheat, presenting a major obstacle to achieving the goal of sustainably providing enough yield to feed the world. The wheat stem sawfly (WSS; Cephus cinctus Norton) is a major pest of winter and spring hexaploid wheat and tetraploid durum wheat (T. turgidum ssp. durum), which are harvested across the Northern Great Plains of North America (Lesieur et al. 2016).

WSS completes its life cycle within a year, most of which is spent in the larval stages. The adult first emerge from the 
previous year's stub in early summer, typically in mid to late June (Beres et al. 2011b; Nilsen et al. 2016). Female adults, which live for 7-10 days, lay their eggs inside the wheat stem, and the resulting larvae begin feeding on the parenchyma and vascular tissues. Following the fifth instar stage, the larvae move down the inside of the stem and cut the stem at the base. The toppled stem provides shelter for the larvae, allowing them to overwinter during the obligatory diapause stage (Beres et al. 2011a). Heavy WSS infestation can cause up to $95 \%$ of the stems to lodge due to this stem-cutting behavior (Beres et al. 2011b). The combination of lodging and decreased photoassimilation due to vascular injury may account for yield losses as high as $30 \%$, equating to economic losses of $\$ 350$ million annually (Delaney et al. 2010; Beres et al. 2011a; Nilsen et al. 2016). Since the larvae are protected inside the stems and the adult flies do not feed, chemical control strategies against WSS are ineffective. Insecticides can instead affect the natural enemies of WSS, impeding the biological control of this pest. Cultural practices offer limited benefits and often involve additional costs. WSS management strategies have therefore conventionally relied on host plant resistance (Beres et al. 2011b).

A key trait in host plant resistance to WSS is the pith-filled stem structure that reduces larval survival (Beres et al. 2011a, b; Buteler et al. 2015) and deters oviposition (Varella et al. 2017). Recently, long noncoding RNAs in wheat and microRNAs (miRNAs) in WSS larva were implicated in wheat resistance to infestation and the suppression of this resistance, respectively (Cagirici et al. 2017a). A major locus on chromosome 3BL, Qss.msub-3BL, was found to account for at least $76 \%$ of the total variation in stem solidness, and other minor alleles located on chromosomes 1B, 3D, and 5D have also been identified in hexaploid wheat (Nilsen et al. 2016; Varella et al. 2015). A single dominant gene, SSt1, controlling stem solidness in durum wheat, has been mapped to chromosome 3BL, in the region of the Qss.msub-3BL locus (Nilsen et al. 2016). Despite several studies aiming to map the loci responsible for the solid stem phenotype, the underlying molecular mechanisms contributing to this key trait remain elusive.

The annotation and assembly of transcripts from WSS larva and male and female adults revealed the main gene classes involved in ion-binding and biosynthetic processes (Cagirici et al. 2017a). The cross-kingdom regulation of miRNAs was also elucidated to assess the possible effects of larval miRNAs on wheat. A total of 10 putative wheat targets for three larval miRNAs were reported on wheat chromosome 3 , including regions encoding the probable methyltransferase PMT11 and ankyrin-like proteins (Cagirici et al. 2017a). These putative wheat targets of larval miRNAs therefore likely function in the wheat defense mechanisms against WSS.

In this study, we used a multiomics approach in the solidand semi-solid-stemmed hexaploid wheat cultivars, Choteau and Scholar, respectively, to better understand the underlying molecular mechanisms of the WSS response and the contribution of stem solidness to WSS resistance. We also conducted a comparative genomics analysis of the stem solidness loci in the wild emmer (T. turgidum ssp. dicoccoides), durum, and bread wheats, and analyzed the syntenic relationship of the 3BL locus in three close relatives of wheat: barley (Hordeum vulgare), Brachypodium (Brachypodium distachyon), and rice (Oryza sativa). Furthermore, we conducted an in silico miRNA identification for 3BL to gain further insights into the regulatory mechanisms that could contribute to stem solidness and WSS resistance. Taken together, this study represents the first report of the advantages of using comparative genomics, transcriptomics, proteomics, and metabolomics approaches to provide a comprehensive understanding of wheat defense mechanisms against WSS, and paves the way for future improvements in WSS management.

\section{Materials and methods}

\section{Datasets used in this study}

Molecular markers associated with stem solidness were retrieved from previous reports (Nilsen et al. 2017), and marker sequences were retrieved from either TriticeaeToolbox (https:// triticeaetoolbox.org/) or GrainGenes (http://wheat.pw.usda.gov/ GG3/). The 3B chromosome sequences and transcript annotations used were from wild emmer wheat (T. turgidum ssp. dicoccoides genotype "Zavitan"; Avni et al. 2017) and the $3 \mathrm{~B}$ chromosome assemblies, or pseudomolecules, and transcript annotations were from bread wheat (T. aestivum cv. Chinese Spring; IWGSC 2014; Choulet et al. 2014).

\section{Plant material and WSS treatment}

In this study, stem tissues from two wheat cultivars, the solidstemmed "Choteau" (PI633974) and semi-solid-stemmed "Scholar" (PI607557), were used. These lines carry distinct alleles for solid stems at Qss.msub-3BL. All plants were grown in the greenhouse under a 16-h photoperiod. Adult WSS (Cephus cinctus) were obtained for the experiments. Three plants per pot were grown with daily watering, incorporating twice-a-week fertilizing after the three-leaf stage. At Zadok stage 32 (two detectable internodes), the plants were individually placed inside infestation chambers containing three adult female WSS for 3-4 days. Control plants were caged but not exposed to WSS. Plant tissues were sampled 14 days after the initial infestation date; first, the lower internodes of the stem were dissected to identify infestation with either eggs or larvae, then the plant material was collected solely from internodes 3 and 4 , at the top of the region containing mature tissues. The same stem section was collected from the control plants. Stem material from infested and 
control plants was immediately flash-frozen in liquid nitrogen and stored at $-80{ }^{\circ} \mathrm{C}$.

\section{Total RNA isolation, sequencing, and identification of differentially expressed genes}

Total RNA was extracted for three replicates of control and infested stem tissues from the two cultivars, as previously described (Cagirici et al. 2017a). RNA sequencing (RNASeq) was performed on the isolated RNA samples with RNA integrity numbers of greater than 7. The library was prepared using a TruSeq RNA Library Preparation Kit (Illumina, San Diego, CA, USA), following the manufacturer's instructions, and sequencing was performed using an Illumina Hi-Seq 2000 platform. The RNA-Seq reads were trimmed using Sickle (v.1.33) before being aligned to the $T$. aestivum chromosome 3B assembly using GMAP (v.201702-25; Wu and Watanabe 2005). The aligned reads were then compared to a GFF file containing annotated transcripts of $T$. aestivum (variety Chinese Spring) and the read counts were obtained using HTSeq-count (-m union; Anders et al. 2015). Raw read counts were filtered using the Noleaven $\mathrm{R}$ package (https://github.com/topherconley/noleaven) to eliminate those with zero or low counts across the entire dataset. The identification and functional annotation of differentially expressed genes (DEGs) between the control and WSStreated stem samples of Choteau and Scholar were performed as previously described (Cagirici et al. 2017a).

\section{Analysis of proteome changes in response to WSS infestation}

The total proteins of three replicates of the control and infested stem tissues of Choteau and Scholar were extracted using the TCA-phenol method (Wang et al. 2006) and quantified as described previously (Esen 1978). The protein extracts were denatured by the addition of lysis buffer (1:1) containing $7 \mathrm{M}$ urea, $2 \mathrm{M}$ thiourea, and 4\% 3-((3-cholamidopropyl) dimethyl ammonio)-1-propanesulfonate (CHAPS), followed by the addition of $30 \mathrm{mM}$ Tris- $\mathrm{HCl}$ ( $\mathrm{pH}$ 8.8). Subsequently, each set of three samples, labeled with a CyDye dilution of $\mathrm{Cy} 2, \mathrm{Cy} 3$, or Cy5 (GE Healthcare Biosciences, Pittsburgh, PA, USA), was run on a single gel. The labeling was stopped by the addition of $0.7 \mu \mathrm{L}$ L-lysine $(10 \mathrm{mM})$ followed by a 15 -min incubation at $4{ }^{\circ} \mathrm{C}$. The labeled samples were mixed with an equal volume of $2 \times 2$ D sample buffer ( $8 \mathrm{M}$ urea, $4 \%$ CHAPS, $20 \mathrm{mg} /$ $\mathrm{mL}$ dithiothreitol (DTT), 2\% Pharmalytes (GE Healthcare Biosciences), and a trace amount of bromophenol blue) and $100 \mu \mathrm{L}$ destreak solution (GE Healthcare Biosciences). The total sample volumes were adjusted to $260 \mu \mathrm{L}$ with rehydration buffer (7 M urea, $2 \mathrm{M}$ thiourea, $4 \%$ CHAPS, $20 \mathrm{mg} / \mathrm{mL}$ DTT, $1 \%$ Pharmalytes, and a trace amount of bromophenol blue). The samples were analyzed for isoelectric focusing on a 13-cm precast non-linear immobilized $\mathrm{pH}$ gradient strip $(\mathrm{pH}$ 4-9; GE Healthcare Biosciences) and separated for size in the second dimension using sodium dodecyl sulfate polyacrylamide gel electrophoresis. The gels were scanned using a Typhoon Trioscanner (GE Healthcare Biosciences) following the manufacturer's protocol, and the gel images were processed with Image Quant (v.5.0; GE Healthcare Biosciences).

The differential protein levels were quantified using a differential in-gel analysis, and a quantitative analysis of the protein spots was performed using DeCyder software (v.6.5; GE Healthcare Biosciences). Quantitative comparisons of spots were performed for samples run at the same time, and the pair-wise volume ratios were calculated for each protein spot to determine the relative protein levels. A Student's $t$ test was conducted using the $\log _{2}$-normalized average spot volume ratios for all spots detected from the three replicates of each experiment. Only spots representing differentially regulated proteins with $\mathrm{a} \geq 1.35$-fold and statistically significant ( $p$ value $<0.05$ ) difference were selected for mass spectrometry (MS). The selected spots were subjected to in-gel trypsin digestion, peptide extraction, desalting, and spotting on a matrix-assisted laser desorption/ionization (MALDI) plate, followed by a MALDI time-of-flight (TOF) analysis for protein identification. The mass spectra of the peptides in each sample were obtained using an Applied Biosystems Proteomics Analyzer (Thermo Fisher Scientific, Waltham, MA, USA), and the 10-20 most abundant peptides in each sample were further subjected to fragmentation and a tandem MS/MS analysis. Combined results from the MS and MS/MS spectra were submitted for a primary sequence database search using the GPS Explorer software equipped with the MASCOT search engine to identify the proteins. The highest scoring hit from the database search for each 2D gel spot was used as the protein identification label. Candidates with a protein score confidence interval (C.I.) or ion C.I. of $>95 \%$ were considered significantly DEGs.

\section{Analysis of metabolome changes in response to WSS infestation}

Total metabolite extraction was performed for three replicates of control and infested stem tissues of Choteau and Scholar. Approximately $150 \mathrm{~g}$ of the frozen stem sample was ground in liquid nitrogen and immersed in $100 \%$ methanol at $70{ }^{\circ} \mathrm{C}$ for $15 \mathrm{~min}$. Subsequently, the samples were vortexed and centrifuged at $25,000 \mathrm{~g}$ for $10 \mathrm{~min}$ at $4{ }^{\circ} \mathrm{C}$. The metabolites were separated by acetone precipitation at $-80^{\circ} \mathrm{C}$ with overnight incubation, followed by centrifugation at $25,000 \mathrm{~g}$ for $10 \mathrm{~min}$ at $4{ }^{\circ} \mathrm{C}$. The resulting metabolite supernatant was dried in a speed vacuum and stored at $-80{ }^{\circ} \mathrm{C}$.

Following the method of Fiehn et al. (2008), the metabolites were separated using gas chromatography (GS)-MS, using helium as the mobile phase with a flow rate of $1 \mathrm{~mL} \mathrm{m^{-1 }}$, on a Rtx-5Sil MS column (30 m length $\times$ 
$0.25 \mathrm{~mm}$ internal diameter with a $0.25-\mu \mathrm{m}$ film of $95 \%$ dimethyl/5\% diphenylpolysiloxane; Restek Corporation, Bellefonte, PA, USA). The injection temperature was $50^{\circ} \mathrm{C}$, which ramped to $250^{\circ} \mathrm{C}$ by $12{ }^{\circ} \mathrm{C} \mathrm{s}^{-1}$. The initial oven temperature was $50{ }^{\circ} \mathrm{C}$ for $1 \mathrm{~min}$, which was then ramped at $20{ }^{\circ} \mathrm{C} \mathrm{min}{ }^{-1}$ to $330{ }^{\circ} \mathrm{C}$ and held constant for $5 \mathrm{~min}$. Following the GC-TOF MS, the primary metabolites were profiled and their abundance was estimated based on peak intensity. Scaling and centering of the data were performed following the method suggested by van den Berg et al. (2006). Metabolites that are produced by the pathways suggested to be differentially regulated under WSS infestation by the transcriptomic and proteomic data were analyzed further.

\section{In silico miRNA identification, target prediction, and annotation}

A total of 1404 non-redundant high-confidence and/or experimentally identified mature miRNA sequences from 72 Viridiplantae species were collected from miRBase (v.21, June 2014; Kozomara and Griffiths-Jones 2011). A two-step homology-based in silico method was then used to identify miRNAs from the transcriptome sequences detailed above, as previously described (Akpinar et al. 2015; Alptekin and Budak 2017; Cagirici et al. 2017b; Kantar et al. 2012; Kurtoglu et al. 2014). Potential mRNA targets of the miRNAs were identified with user-defined query and target options defined by Dai and Zhao (2011).

\section{Results}

\section{Comparative genomics analysis of stem solidness loci in wild emmer, durum, and bread wheats}

Stem solidness, characterized by pith formation inside the stem that serves as a physical barrier against larval movement, is considered to be the most important trait in the integrated pest management of WSS. Although this complex trait appears to be under the control of several genes on different chromosomes, the Qss.msub-3BL locus, located on the long arm of chromosome 3B, accounts for most of the variation in wheat stem solidness (Kong et al. 2013; Nilsen et al. 2017). To explore the physical locations of the genetic determinants of stem solidness, including the Qss.msub-3BL locus, previously published molecular markers linked to this trait (Nilsen et al. 2017) were mapped to the chromosome $3 B$ pseudomolecules of T. turgidum ssp. dicoccoides genotype Zavitan (wild emmer wheat), T. turgidum ssp. durum genotype MWG (tetraploid durum wheat), and T. aestivum cv. Chinese Spring (hexaploid wheat; Fig. 1). Wild emmer wheat is a close wild relative of durum wheat, as both diverged from the common ancestor $T$. turgidum, the tetraploid progenitor of T. aestivum (Ani
Akpinar et al. 2015). The close relationship between the three wheat genomes appears to be retained for the molecular markers associated with the stem solidness trait (Fig. 1).

The physical location of the molecular markers linked to stem solidness identified six regions associated with this trait along chromosome 3B. The first region was located on the short arm, while the remaining five loci were clustered closer to the distal end of the long arm of chromosome 3B. Besides these regions, five additional markers were mapped to separate locations. Curiously, two of these markers suggested that an additional locus exists in durum wheat within a cluster of 11 molecular markers. Although the molecular markers indicated a scattered positioning of genetic determinants for stem solidness, most were clustered specifically at the distal-most region of chromosome 3B (Fig. 2). This region, consistent with previous reports (Nilsen et al. 2017), likely represents the Qss.msub-3BL locus, and is located between $809.2-829.2 \mathrm{Mb}$ (i.e., between markers Kukri_c86043_197 and BS00065603) in hexaploid wheat, $823-841.1 \mathrm{Mb}$ (Tdurum_contig12530_278 and Tdurum_contig59566_4435) in emmer wheat, and 816.3$836.2 \mathrm{Mb} \quad(\mathrm{Kukri}$ c $86043 \ldots 197$ and Tdurum_contig59566_4435) in durum wheat. This locus is hereafter referred to as $Q s s-3 \mathrm{BL}$.

\section{Syntenic analysis of Qss-3BL in barley, Brachypodium, and rice}

No information on the conservation of the well-known wheat Qss-3BL quantitative trait locus (QTL) is available in barley, Brachypodium, and rice, close relatives of wheat, despite the threat that WSS poses to barley production (Portman et al. 2016). This is not surprising, as a comparison of the molecular markers from the $Q s s-3 B L$ QTL with the syntenic Brachypodium chromosome $2(\mathrm{Bd} 2)$ and rice chromosome 1 (Os1) indicated little conservation at the nucleotide level. Thus, to explore the syntenic relationships between these closely related species, wheat transcript sequences mapped to the $Q s s-3 B L$ interval were compared against the annotated proteins from barley, Brachypodium, and rice. A best reciprocal hit strategy was used, with the sequence similarity cutoff for significant pairs adjusted for barley, the closest wheat relative of the three. This comparison identified orthologous $Q s s-3 B L$ genes in the syntenic chromosomes $\mathrm{Bd} 2$, Os1, and the long arm of chromosome $3 \mathrm{H}$ in barley (Hv3HL). While there was extensive colinearity between the wheat Qss-3BL QTL and barley $\mathrm{Hv} 3 \mathrm{HL}$, the syntenic regions on $\mathrm{Bd} 2$ and Os1 indicated a few rearrangements (Fig. 3a). The syntenic regions on $\mathrm{Bd} 2$ appeared to span between Bradi2g59970 and Bradi2g62670, encompassing 40 genes, although orthologous genes were identified on more distant regions as well, such as Bradi2g04010. The syntenic block on Os1 was defined by 42 genes located between Os01g0964900 and Os01g0976900, although, similar to Brachypodium, more orthologous genes were found in other 
Tae3BL

Tdu3BL

Tdic3BL

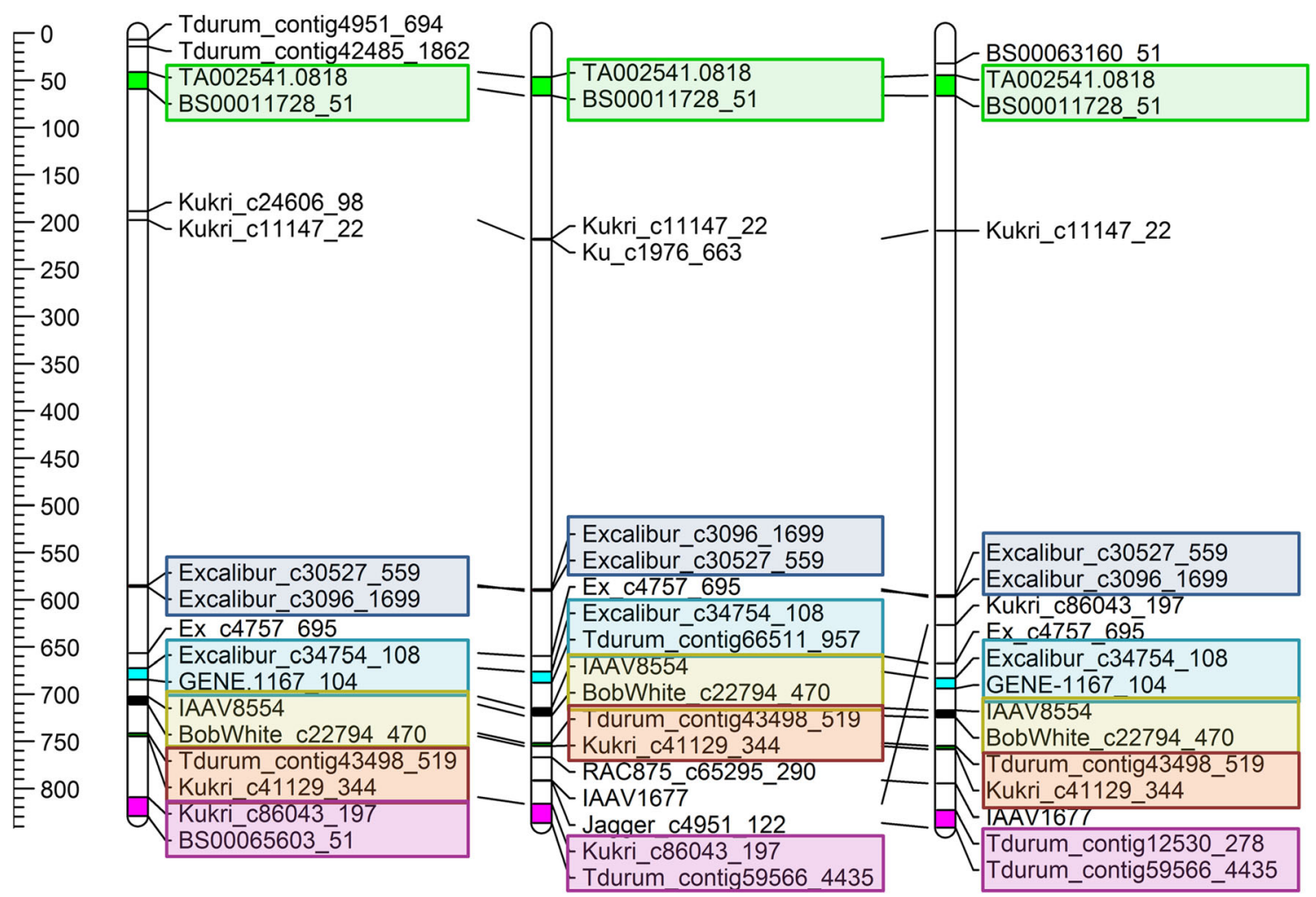

Fig. 1 Comparative analysis of the regions delineated by the molecular markers linked to stem solidness on the $3 B$ chromosomes of T. aestivum, T. turgidum durum, and T. turgidum dicoccoides. The six regions identified by the molecular markers are indicated by different colors.
Tae, Triticum aestivum cultivar Chinese Spring; Tdu,: Triticum turgidum durum accession MWG; Tdic, Triticum turgidum dicoccoides accession Zavitan
Tae3BL

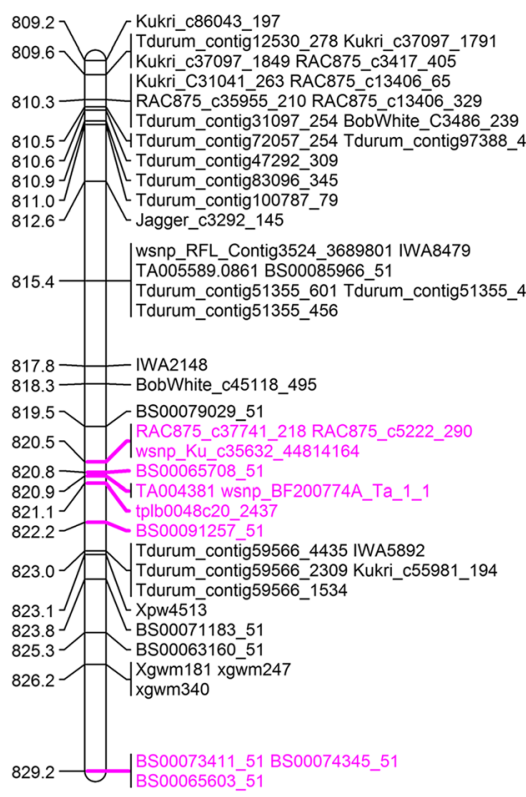

Tdu3BL

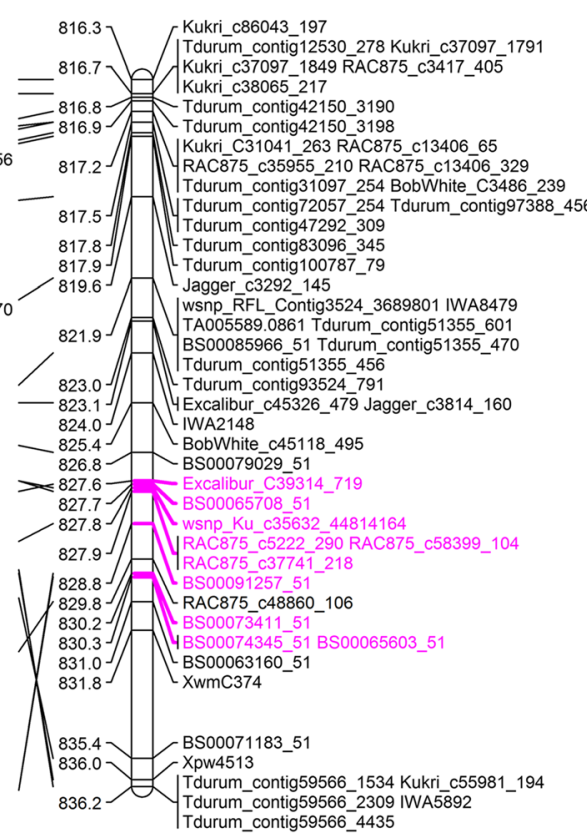

Tdic3BL

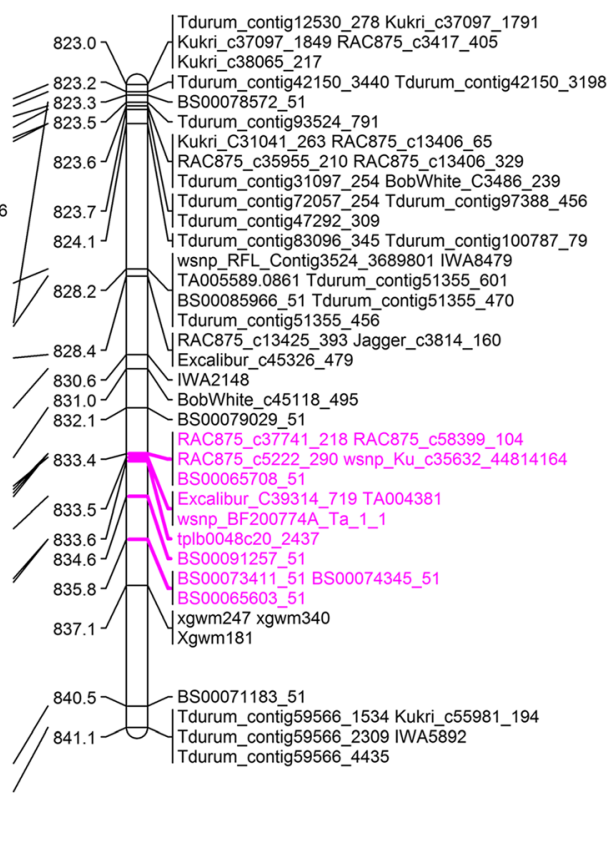

Fig. 2 Comparative analysis of the Qss-3BL QTL associated with WSS resistance in T. aestivum, T. turgidum durum, and T. turgidum dicoccoides, indicated by molecular markers. Pink shading indicates the most probable location of the SSt1 gene found in tetraploid wheat.
Tae, Triticum aestivum cultivar Chinese Spring; Tdu, Triticum turgidum durum accession MWG; Tdic, Triticum turgidum dicoccoides accession Zavitan 
regions of the chromosome. In barley, the syntenic interval was composed of 44 highly conserved genes between HORVU3Hr1G112590 and HORVU3Hr1G117860, in an almost identical order to the orthologous genes in the wheat Qss-3BL locus (Fig. 3a). The high level of conservation for these loci between the 3BL and 3HL chromosomes demonstrates the close evolutionary relationship between wheat and barley. The functional annotations of the Brachypodium, rice, and barley genes located on the putative WSS response loci were strikingly similar to the wheat genes (Supplementary Table S1). This observation suggests that, in addition to their considerable colinearity, these loci may also be functionally conserved among the grasses.

Recently, the SSt1 gene, widely accepted as the basis for stem solidness in tetraploid wheat, was reported to be located between 833.4 and $835 \mathrm{Mb}$ on emmer wheat chromosome 3B (Nilsen et al. 2017). A comparative transcriptomic analysis of the $Q s s-3 B L$ locus between hexaploid bread wheat and the tetraploid wild emmer and durum wheats indicated that the transcripts located in this QTL are highly colinear (Fig. 3b);

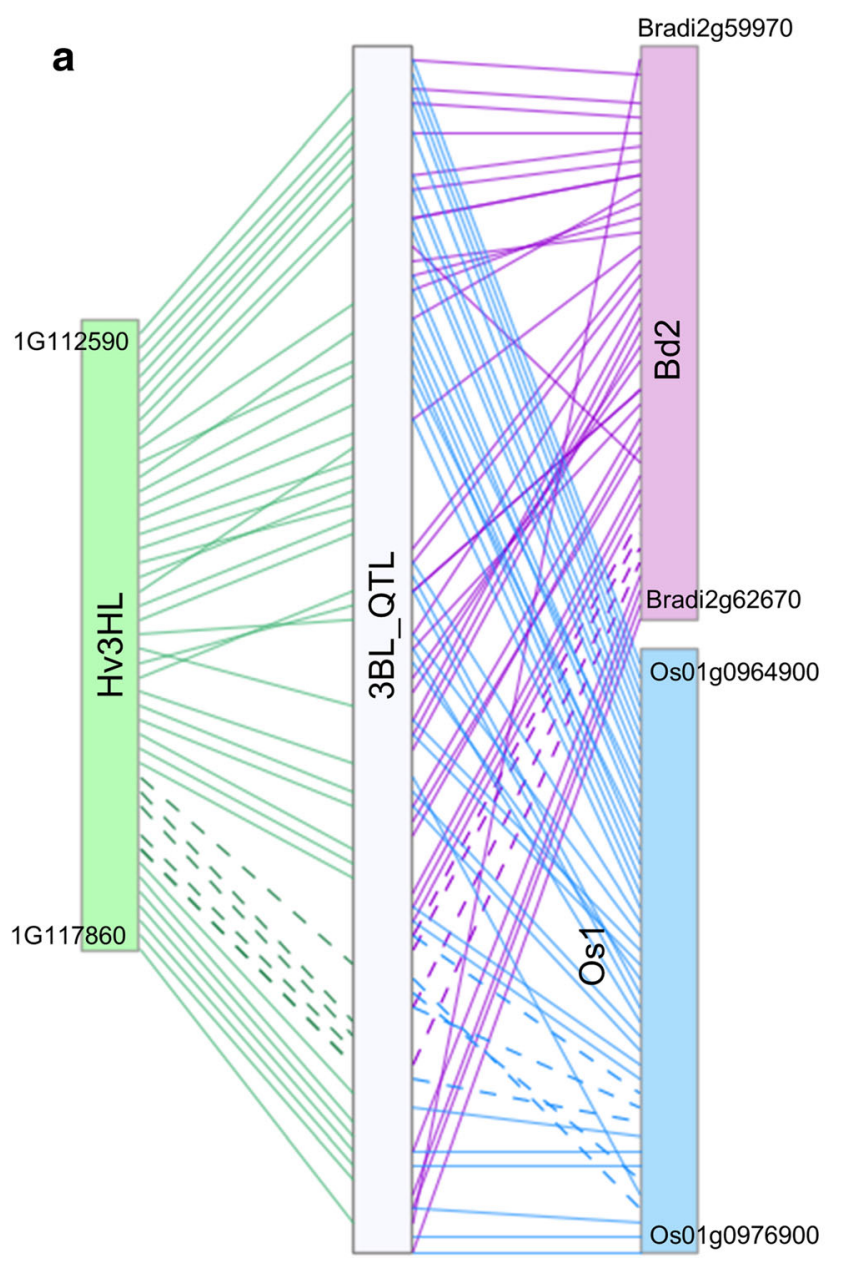

Fig. 3 Chromosomal view of the syntenic relationships of the WSS response locus and the regulatory miRNAs encoded in the Qss-3BL QTL. a Locations of Qss-3BL QTL in Brachypodium distachyon chromosome 2 (Bd2), Oryza sativa chromosome 1 (Os1), and Hordeum however, the putative $S S t 1$ interval within the $Q s s-3 B L$ QTL appears to be split in the hexaploid bread wheat, and the transcripts between these split fragments had no significant counterparts in wild emmer wheat (Fig. 3b, dashed lines). The SSt 1 gene therefore appears to be confined to the tetraploid genotypes.

\section{miRNAs processed from the Qss-3BL locus}

Putative miRNAs encoded from the distal-most loci delineated by the $Q s s-3 B L$ molecular markers on chromosome 3B of bread, durum, and wild emmer wheats, including the putative SSt1 gene reported in previous study (Nilsen et al. 2017), were explored using a two-step homology-based optimized method (Lucas and Budak 2012; Alptekin et al. 2016). The comparison of these regions to the 1404 high-confidence mature miRNA sequences of plants retrieved from the miRBase database (Release 21) indicated that these three loci from bread, durum, and wild emmer wheats carried precursor sequences for 454 , 451 , and 447 unique mature miRNAs belonging to 17,18 , and

b

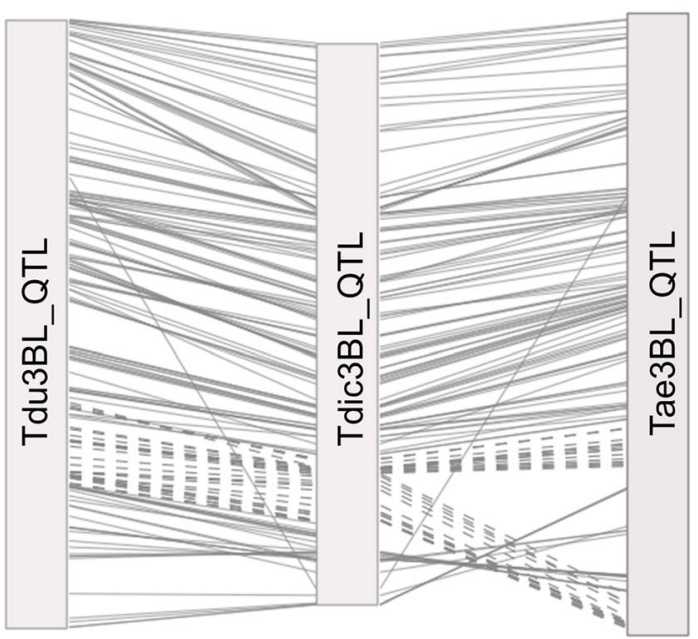

C

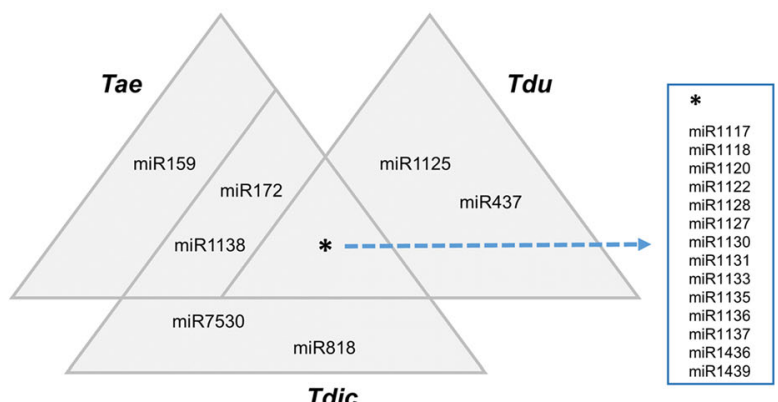

vulgare chromosome 3 (Hv3H). b T. aestivum, T. turgidum durum, and T. turgidum dicoccoides. c miRNAs identified from the Qss-3BL QTL of T. aestivum, T. turgidum durum, and T. turgidum dicoccoides 
16 families, respectively (Fig. 3c). Of these miRNA families, the miR159 family was only identified in bread wheat, miR1125 and miR437 were only found in durum wheat, while miR7530 and miR818 were specific to loci on the wild emmer wheat $3 \mathrm{~B}$ chromosome. Furthermore, miR1139 and miR172 were only shared by the bread and durum wheat loci on the $3 \mathrm{~B}$ chromosomes, not the wild emmer wheat. The remaining 14 miRNA families were common in all three species (Budak et al. 2015).

Plant miRNAs require near-perfect complementation with their target transcripts, enabling the robust identification of potential targets for a given miRNA (Budak and Akpinar 2015). All candidate mature miRNAs were compared against high-confidence coding sequences from bread, durum, and wild emmer wheats to explore the potential miRNA-target pairs and possible networks behind the regulation of the WSS response. miRNAs stemming from the $Q s s-3 B L$ locus in bread wheat targeted 122 coding transcripts, only one of which mapped to the same region. Notably, this single transcript was involved in miRNA-target pairing with 23 unique mature miRNA isoforms from the miR1127 family, even though it did not appear among the DEGs between Choteau and Scholar under control conditions and WSS infestation. Interestingly, 83 mature miRNAs were found to potentially regulate the expression of seven transcripts on the $Q s s-3 B L$ locus believed to encode disease resistance-related proteins. A total of 632 transcripts were potentially targeted by 337 unique mature miRNAs, although only three of them mapped onto the interval potentially containing SSt1 on durum wheat chromosome 3B. Similar to the transcripts targeted by the bread wheat miRNAs, seven durum wheat transcripts targeted by the miRNAs potentially encoded disease resistance-related proteins. None of the 292 coding targets of wild emmer wheat miRNAs were transcribed from the WSS response locus, whereas four genes encoding disease-resistance proteins in other genomic regions were targeted by four of the $Q s s-3 B L$ miRNA families.

\section{Differentially regulated transcripts in response to WSS infestation}

To gain further insights into how the six 3B loci associated with stem solidness contribute to this trait, RNA-Seq was performed on the semi-solid and solid stem tissues of two bread wheat cultivars, Scholar and Choteau, with and without WSS infestation. In total, 36 transcripts from the six loci were differentially regulated in the different genotypes and/or in response to WSS infestation ( $p$ value $<0.05$ ).

To identify the differentially regulated transcripts, clean RNA-Seq reads were mapped onto the T. aestivum Chinese Spring RefSeq v. 1 chromosome 3B pseudomolecule. In general, a trend towards downregulation was observed, including for potentially stress-related transcripts (Table 1). The semisolid-stemmed cultivar Scholar exhibited the differential regulation of 15 transcripts in response to WSS infestation, of which only five were upregulated in comparison with the control conditions. Transcripts encoding components of protein metabolism and transport, including an AP-3 complex subunit beta- 2 and an F-box domain-containing protein, as well as multiple peptidases, were downregulated in the infested Scholar stem tissues. In addition, two leucine-rich repeat (LRR) domain-containing proteins, one of which contained a NB-ARC domain, were also downregulated. By contrast, auxin signaling may be promoted in response to WSS infestation, as indicated by the upregulation of a transcript encoding an auxin efflux carrier component. Other transcripts upregulated in response to WSS infestation included those encoding the biotic stress-related constitutive expressor of pathogenesis-related (PR) genes 5 (CPR-5) protein, NADH dehydrogenase (NDH-A), and a magnesium transporter (Table 1).

The solid-stemmed variety Choteau had nine differentially expressed genes upon WSS infestation, of which only one, encoding a cysteine-rich receptor-like protein kinase, was upregulated. Transcripts potentially encoding stress-related proteins and components of signal transduction, including the receptor-like protein kinase FERONIA, a rhodanese-like domain-containing protein, a salt stress response/antifungal domain-containing protein kinase, an LRR protein kinase, and a wall-associated receptor kinase, were strongly downregulated in Choteau stems in response to WSS infestation (Table 1).

While the comparison of control and infested samples provides insights into the molecular mechanisms selectively activated in response to WSS infestation, a comparison of cultivars with varying levels of stem solidness under both control and infestation conditions may reveal the specific phenotype that restricts WSS larval growth, i.e., stem solidness. A comparison of the Choteau and Scholar stem tissues in control conditions revealed seven transcripts that were differently regulated between these cultivars. Under normal growth conditions, the stem-tissue expression levels of genes encoding an LRR protein kinase, a thaumatin domain-containing protein kinase, and a KH-domain-containing protein were up to twice as high in Choteau than in Scholar (Table 1). By contrast, a transcript encoding a Domain of Unknown Function (DUF)containing protein and two transcripts with no homology to any known Viridiplantae proteins were expressed between 2.7- and 4.8-fold higher in Scholar than Choteau stems.

In response to WSS infestation, the expression levels of transcripts encoding a threonyl-tRNA synthetase and an AP3 complex subunit beta- 2 were higher in Choteau stems than in Scholar, as these transcripts were strongly downregulated in the Scholar stem tissue upon infestation (Table 1). Another potentially stress-related transcript, encoding phosphoglycerate mutase, was also found at higher levels in infested Choteau stem samples. By contrast, the transcripts for the receptor-like 
Table 1 Differentially regulated transcripts between semi-solid-stemmed Scholar and solid-stemmed Choteau wheat cultivars, with and without WSS infestation

\begin{tabular}{|c|c|c|c|c|c|c|c|c|c|c|}
\hline \multirow[b]{2}{*}{ Transcript } & \multirow[b]{2}{*}{$\begin{array}{l}\text { Location }^{\mathrm{a}} \\
\text { (bp) }\end{array}$} & \multirow[b]{2}{*}{ Protein product } & \multicolumn{2}{|c|}{$\begin{array}{l}\text { ChoInf vs. } \\
\text { ChoWT }\end{array}$} & \multicolumn{2}{|c|}{$\begin{array}{l}\text { SchInf vs. } \\
\text { SchWT }\end{array}$} & \multicolumn{2}{|c|}{$\begin{array}{l}\text { ChoWT vs. } \\
\text { SchWT }\end{array}$} & \multicolumn{2}{|c|}{$\begin{array}{l}\text { ChoInf vs. } \\
\text { SchInf }\end{array}$} \\
\hline & & & $\begin{array}{l}\log \\
\text { FC }\end{array}$ & $p$ val & $\begin{array}{l}\log \\
\text { FC }\end{array}$ & $p$ val & $\begin{array}{l}\log \\
\text { FC }\end{array}$ & $p$ val & $\begin{array}{l}\log \\
\text { FC }\end{array}$ & $p$ val \\
\hline TraesCS3B01G069400 & $41,351,591$ & $\begin{array}{l}\text { Domain of Unknown Function } \\
\text { (DUF1618)-containing protein }\end{array}$ & - & - & - & - & -4.8 & 0.020 & - & - \\
\hline TraesCS3B01G069500 & $41,426,379$ & - & - & - & - & - & -2.7 & 0.044 & - & - \\
\hline TraesCS3B01G072700 & $43,832,324$ & $\begin{array}{l}\text { Putative LRR receptor-like serine/threonine- } \\
\text { protein kinase }\end{array}$ & - & - & - & - & 2.0 & 0.034 & - & - \\
\hline TraesCS3B01G074900 & $46,007,493$ & - & - & - & - & - & - & - & 3.3 & 0.003 \\
\hline TraesCS3B01G076900 & $47,452,833$ & Thaumatin domain-containing protein kinase & - & - & - & - & 1.8 & 0.019 & - & - \\
\hline TraesCS3B01G080500 & $50,932,437$ & Threonyl-tRNA synthetase & - & - & -5.6 & 0.012 & - & - & 5.7 & 0.012 \\
\hline TraesCS3B01G086500 & $54,784,828$ & Receptor-like protein kinase FERONIA & -5.1 & 0.010 & - & - & - & - & -4.5 & 0.025 \\
\hline TraesCS3B01G088200 & $56,128,728$ & Putative disease-resistance protein RGA3 & - & - & - & - & - & - & -5.0 & 0.031 \\
\hline TraesCS3B01G373000 & $585,829,361$ & Metalloenzyme superfamily protein/p & - & - & - & - & - & - & 1.4 & 0.011 \\
\hline TraesCS3B01G435600 & $673,849,549$ & Rhodanese-like domain-containing protein & -3.0 & 0.047 & - & - & - & - & -3.0 & 0.046 \\
\hline TraesCS3B01G438500 & $677,740,856$ & - & - & - & - & - & -4.8 & 0.022 & - & - \\
\hline TraesCS3B01G440800 & $680,420,121$ & Probable magnesium transporter & - & - & 2.7 & 0.025 & - & - & - & - \\
\hline TraesCS3B01G458900 & $702,682,149$ & LRR-domain-containing protein tyrosine kinase & - & - & -3.4 & 0.025 & - & - & - & - \\
\hline TraesCS3B01G461200 & $704,324,107$ & CPR-5-like protein & - & - & 1.2 & 0.043 & - & - & - & - \\
\hline TraesCS3B01G462800 & $705,699,031$ & alpha/beta hydrolase-fold-containing protein & - & - & - & - & - & - & -1.6 & 0.034 \\
\hline TraesCS3B01G462900 & $705,702,223$ & $\begin{array}{l}\text { Auxin efflux carrier component, transmembrane } \\
\text { protein }\end{array}$ & - & - & 1.5 & 0.009 & - & - & - & - \\
\hline TraesCS3B01G466500 & $708,950,606$ & $\begin{array}{l}\text { Arabinosyltransferase ARAD1/exostosin } \\
\text { domain-containing protein }\end{array}$ & - & - & - & - & - & - & -4.3 & 0.035 \\
\hline TraesCS3B01G499400 & $744,228,112$ & - & - & - & -3.8 & 0.003 & - & - & - & - \\
\hline TraesCS3B01G580400 & $809,479,669$ & $\begin{array}{l}\text { Salt stress response/antifungal domain- } \\
\text { containing protein kinase }\end{array}$ & -4.5 & 0.030 & - & - & - & - & - & - \\
\hline TraesCS3B01G580700 & $809,602,487$ & $\mathrm{KH}$ domain-containing protein & - & - & - & - & 1.8 & 0.040 & - & - \\
\hline TraesCS3B01G580900 & $809,625,786$ & - & -3.1 & 0.045 & -4.2 & 0.006 & - & - & - & - \\
\hline TraesCS3B01G582100 & $810,450,715$ & Retrotransposon-related protein & - & - & - & - & 2.6 & 0.005 & - & - \\
\hline TraesCS3B01G582200 & $810,472,321$ & NDH-A protein & - & - & 2.1 & 0.017 & - & - & - & - \\
\hline TraesCS3B01G583000 & $810,948,315$ & F-box domain-containing protein & - & - & -3.5 & 0.016 & - & - & - & - \\
\hline TraesCS3B01G584600 & $811,457,387$ & - & - & - & - & - & - & - & -1.6 & 0.004 \\
\hline TraesCS3B01G585600 & $812,569,184$ & $\begin{array}{l}\text { Aminopeptidase I zinc metalloprotease } \\
\text { domain-containing protein }\end{array}$ & - & - & 1.9 & 0.031 & - & - & - & - \\
\hline TraesCS3B01G588300 & $814,446,746$ & LRR-domain-containing protein kinase & -1.8 & 0.023 & - & - & - & - & - & - \\
\hline TraesCS3B01G589000 & $814,699,730$ & Methionine aminopeptidase-like protein & - & - & -2.5 & 0.038 & - & - & - & - \\
\hline TraesCS3B01G591700 & $816,170,822$ & Wall-associated receptor kinase & -4.6 & 0.034 & - & - & - & - & - & - \\
\hline TraesCS3B01G593900 & $817,408,885$ & NB-ARC-LRR domain-containing protein & - & - & -4.6 & 0.031 & - & - & - & - \\
\hline TraesCS3B01G594400 & $817,710,500$ & - & - & - & -5.4 & 0.011 & - & - & - & - \\
\hline TraesCS3B01G596400 & $819,298,156$ & $\begin{array}{l}\text { Cytochrome P450 domain-containing } \\
\text { protein/4-hydroxyphenylacetaldehyde } \\
\text { oxime monooxygenase-like }\end{array}$ & -4.6 & 0.024 & - & - & - & - & - & - \\
\hline TraesCS3B01G597900 & $819,930,084$ & Peptidase C13 family protein & - & - & -2.3 & 0.034 & - & - & - & - \\
\hline TraesCS3B01G600100 & $820,789,324$ & AP-3 complex subunit beta- 2 & - & - & -5.2 & 0.009 & - & - & 4.2 & 0.044 \\
\hline TraesCS3B01G602400 & $822,535,369$ & Cysteine-rich receptor-like protein kinase 27 & 3.6 & 0.026 & - & - & - & - & - & - \\
\hline TraesCS3B01G611600 & $829,273,469$ & Soluble inorganic pyrophosphatase & -5.1 & 0.029 & - & - & - & - & - & - \\
\hline
\end{tabular}

ChoInf, Choteau infested; ChoWT, Choteau wild type; SchInf, Scholar infested; SchWT, Scholar wild type

${ }^{\text {a }}$ Physical position on the T. aestivum 3B pseudomolecule 
protein kinase FERONIA and a rhodanese-like domain-containing protein were less abundant in WSS-infested Choteau than those in Scholar stems, consistent with their downregulation in Choteau stem samples upon WSS infestation. Intriguingly, a transcript encoding the arabinosyltransferase ARAD1, involved in cell wall organization, was more than fourfold scarcer in the WSS-infested Choteau stems than in the infested Scholar stems.

\section{Plant defense response against WSS at the proteome and metabolome levels}

To examine the plant defense response at the proteome level, 2D gel-based proteomics were conducted on stem-tissue samples from Choteau and Scholar. A total of 150 protein spots were detected for both varieties (Fig. 4a), and 21 were differentially regulated under WSS stress and thus selected for protein identification (Fig. 4b, Table 2). Choteau had 10 significantly differently regulated proteins with a $p$ value of $<0.05$ and a fold change of $>1.35$ between the control and infested stems, while Scholar had 15. Four proteins, ureidoglycolate hydrolase (spot no. 77), HMG1/2-like protein (spot no. 149), Ras-related RABB1b (spot no. 131), and glyceraldehyde-3-phosphate dehydrogenase 1 (spot no. 83), were identified as significantly differing between treatments in both varieties ( $p$ value $<0.05$ ). Some of the proteins that were differentially regulated under WSS, such as ribulose bisphosphate carboxylase, were similar to those that were altered in response to abiotic stress,

a

IP3

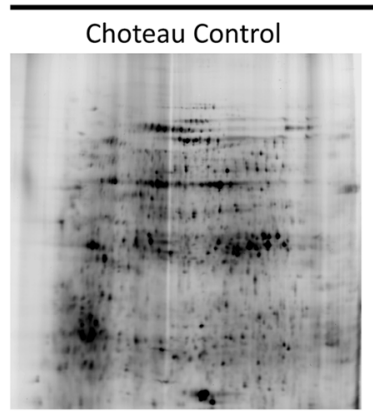

Scholar Control

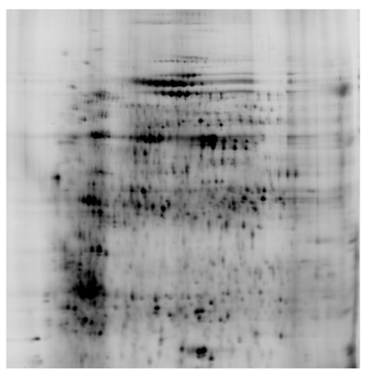

Fig. 4 Proteomics analysis of the WSS response in Scholar and Choteau. a 2D gel photographs of the proteins from the control and WSS-infested stems of Choteau and Scholar. Isoelectronic points (IP) from 3 to 11 and molecular weights (MW) from 150 to $10 \mathrm{kDa}$ are shown on the gel. b suggesting their dual role in both abiotic and biotic stress responses (Budak et al. 2013; Lucas et al. 2011). The identified proteins were analyzed in terms of their association with the stem solidness genomic region; a BLAST analysis was used, where a significant association was defined as more than $50 \%$ of the alignment length and identity. Six of the 21 proteins were significantly mapped to the genomic regions associated with stem solidness, suggesting that these proteins might be encoded by these loci, while the other five proteins did not map to the Qss-3BL region (Table 2). Other proteins showed a level of association to the stem solidness genome regions; however, more research is needed to determine the exact genomic location encoding these proteins.

For Choteau and Scholar, respectively, only three of 10 and four of the 15 differentially abundant proteins were downregulated during the WSS treatment, suggesting a general upregulation in protein expression under WSS infestation (Table 2). Across the four significantly differentially produced proteins identified in both varieties, only ureidoglycolate hydrolase (spot no. 77) was downregulated, while the HMG1/2-like protein (spot no. 149), Ras-related RABB1b (spot no. 131), and glyceraldehyde-3-phosphate dehydrogenase 1 (spot no. 83) were upregulated in both varieties.

Based on the differential regulation of the proteins and stem solidness-associated transcripts, the phenylpropanoid and pentose phosphate pathways were determined to be highly responsive to WSS infestation at the metabolic level. The regulation of the primary metabolites associated with these

b

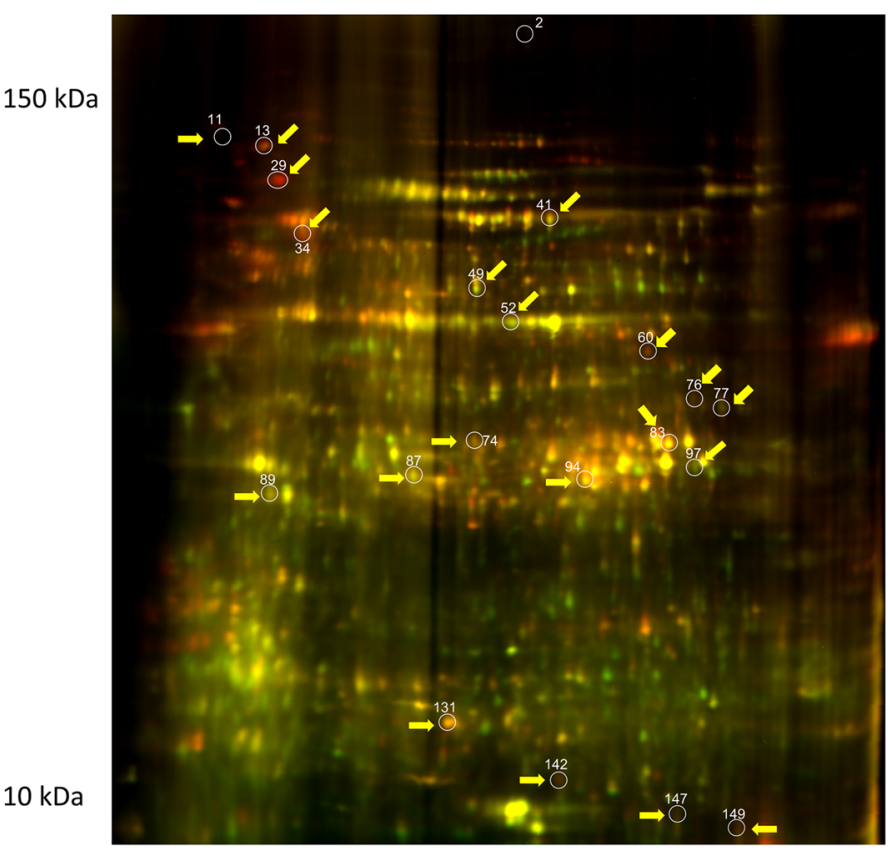

Statistically significant spots ( $p$ value $<0.05$, fold change $>1.35$ ) chosen for mass spectrometry analysis. All spots are indicated by yellow arrows and white circles 
pathways was therefore investigated during WSS infestation using a GC-MS analysis. The metabolomic data indicated that the induced decrease in the amount of the enzyme phenylalanine ammonia-lyase (PAL), the initiator of the phenylpropanoid pathway for secondary metabolite production, during WSS infection affected lignin formation in the wheat, indicated by the slight decrease in lignoceric acid detected in the stem tissues of both Choteau and Scholar (Fig. 5).

By contrast, the metabolites from the pentose phosphate pathway were found to be differently regulated in the solidstemmed Choteau and the semi-solid-stemmed Scholar cultivars. Metabolites associated with the structural sugars arabinose and xylose, which contribute to cell wall biosynthesis, were downregulated in Scholar following WSS infestation, while no significant change was observed in Choteau. Despite the twofold decrease in the amount of fructokinase-2 in Scholar following WSS infestation, there was no significant difference in the abundance of fructose-6-phosphate, the primary compound generated by the activity of this enzyme. The increase in the amount of UDP-glucose 6-dehydrogenase 4 in Scholar did not result in a significant effect on the downstream products of UDP-glucose, such as glucose-1-phosphate and glucose-6phosphate; however, this may be explained by the significant increase in the amount of myo-inositol, which indicated the oxidation of glucose-6-phosphate to generate pentose sugars. In addition, a strong increase in the amount of sucrose-6phosphate was observed in Choteau, which may be associated with an upregulation in sucrose production to provide energy for the plant. By contrast, the levels of both sucrose and sucrose-6-phosphate were decreased in Scholar, which may result in a lack of energy and poor defense against WSS.

\section{Discussion}

Although a major locus (Qss.msub-3BL) and a single dominant gene in durum wheat (SSt1) associated with stem solidness have been identified, in addition to a few minor alleles (Nilsen et al. 2017; Varella et al. 2015), little is known about the molecular mechanisms underlying stem solidness and the alternative types of host plant resistance that may arise from the same locus. Here, we employed a multiomics approach, together with the molecular markers that define the stem solidness-associated locus on chromosome $3 \mathrm{BL}$, to explore the basis of stem solidness and its contribution to WSS resistance, and to investigate the WSS-infestation response in wheat at the molecular level.

\section{Colinearity of the QSS.msub-3BL locus in wheat}

Molecular markers genetically mapped to the Qss.msub-3BL locus in previous reports were clustered around six separate physical regions on the chromosome 3B sequences of three closely related wheat species, hexaploid bread wheat (Chinese Spring), tetraploid durum wheat (MWG), and tetraploid wild emmer wheat (Zavitan). While one of these physical regions was located on the short arm of the chromosome, the remaining five were consecutively located on the long arm in close proximity to each other. Although these six regions were consistently observed on the $3 \mathrm{~B}$ chromosomes of all three wheats, an additional locus was identified on durum wheat chromosome 3B; this 217-219$\mathrm{Mb}$ region consisted of 11 clustered markers, a few of which mapped to independent locations in bread wheat and wild emmer wheat (Fig. 1). Consistent with previous reports on the location of the dominant gene SSt 1 either at or near the 3BL QTL in durum wheat, the molecular markers confining $S S t 1$ to a $\sim 1.6-\mathrm{Mb}$ interval identified by Nilsen et al. (2017) also defined a narrow interval within the most telomeric physical regions of all three species (Fig. 2). These telomeric regions, likely representing the major Qss.msub-3BL locus, are referred to as $Q s s-3 B L$.

Despite close evolutionary relationships, the regions syntenic to the Qss-3BL locus in Brachypodium, rice, and barley have not been accurately defined, likely because of the lack of sequence conservation in the molecular markers, at least between wheat, Brachypodium, and rice. Using a more inclusive approach, a comparison of wheat transcripts located within the $Q s s-3 B L$ locus to the protein sequences of the related grasses clearly identified syntenic regions on the Brachypodium $\mathrm{Bd} 2$ chromosome, the rice Os1 chromosome, and the long arm of the $3 \mathrm{H}$ chromosome in barley (Hv3HL; Fig. 3a). The syntenic intervals indicated small-scale inversions, with few perturbations to the synteny of the independent genes. In contrast to the extensive colinearity in the putative SSt1 region (Fig. 3a, dashed lines) in wheat, barley, and Brachypodium, the corresponding region in rice appears to be rearranged. Importantly, a transcriptomic comparison of the Qss-3BL locus on the 3B chromosomes of the bread, durum, and wild emmer wheats also indicated a disruption in colinearity, specifically in the putative $S S t 1$ region in bread wheat (Fig. 3b, dashed lines). This observation was supported by the fact that $S S t 1$ has only been identified in durum wheat so far (Beres et al. 2013). This split alignment between the bread wheat and wild emmer wheat $Q s s-3 B L$ loci may indicate a rearrangement that occurred after the hybridization of Aegilops tauschii with tetraploid T. turgidum to give rise to the hexaploid T. aestivum, which somehow resulted in the loss of SSt1 in the hexaploid genotypes.

\section{miRNA repertoire of QSS.msub-3BL from wild and modern tetraploid and hexaploid wheats}

Putative miRNAs from the Qss-3BL locus from bread, durum, and wild emmer wheats included 454, 451, and 447 unique mature miRNA sequences belonging to 17,18 , and 16 
Table 2 Differentially regulated proteins between semi-solid-stemmed Scholar and solid-stemmed Choteau wheat cultivars, with and without WSS infestation

\begin{tabular}{|c|c|c|c|c|c|c|c|c|}
\hline \multirow[b]{2}{*}{$\begin{array}{l}\text { Spot } \\
\text { ID }\end{array}$} & \multirow[b]{2}{*}{ Protein name } & \multirow[b]{2}{*}{ NCBI ID } & \multirow[b]{2}{*}{$\begin{array}{l}\text { Protein } \\
\text { MW (kDa) }\end{array}$} & \multirow[b]{2}{*}{$\begin{array}{l}\text { Mapped onto stem } \\
\text { solidness locus }\end{array}$} & \multicolumn{2}{|c|}{$\begin{array}{l}\text { ChoInf/ } \\
\text { ChoWT }\end{array}$} & \multicolumn{2}{|c|}{ SchInf/SchWT } \\
\hline & & & & & $\begin{array}{l}p \\
\text { value }\end{array}$ & $\begin{array}{l}\text { Av. } \\
\text { ratio }\end{array}$ & $p$ value & $\begin{array}{l}\text { Av. } \\
\text { ratio }\end{array}$ \\
\hline 2 & Phenylalanine ammonia-lyase & PAL1_- ORYSJ & 75,451 & Yes, non-significant & 0.045 & -1.66 & 0.49 & -4.37 \\
\hline 60 & Serine hydroxymethyltransferase 4 & $\begin{array}{l}\text { GLYC4 } \\
\text { ARAT- } \\
\text { H }\end{array}$ & 51,685 & No & 0.043 & 2.045 & 0.11 & 1.9 \\
\hline 76 & $\begin{array}{l}\text { 5-methyltetrahydropteroyltriglutamate--homocysteine } \\
\text { methyltransferase } 1\end{array}$ & $\begin{array}{l}\text { METE1- } 1_{-} \\
\text {ORYSJ }\end{array}$ & 84,532 & Yes, non-significant & 0.036 & 1.605 & 0.31 & -1.715 \\
\hline 83 & Glyceraldehyde-3-phosphate dehydrogenase 1, cytosolic & $\begin{array}{l}\text { G3PC1- } \\
\text { HORV- } \\
\quad \mathrm{U}\end{array}$ & 36,491 & Yes, non-significant & 0.026 & 1.465 & 0.029 & 1.83 \\
\hline 97 & Ribulose bisphosphate carboxylase (large chain) & $\begin{array}{l}\text { RBL } \\
\text { HORV- } \\
\text { U }\end{array}$ & 53,045 & Yes, significant & 0.012 & -1.535 & - & - \\
\hline 142 & ATP synthase subunit alpha, mitochondrial & $\begin{array}{l}\text { ATPAM } \\
\text { MAIZE }\end{array}$ & 55,146 & Yes, non-significant & 0.018 & 1.785 & - & - \\
\hline 147 & $\begin{array}{l}\text { 5-methyltetrahydropteroyltriglutamate--homocysteine } \\
\text { methyltransferase } 1\end{array}$ & $\begin{array}{l}\text { METE1 } \\
\text { ORYSJ }\end{array}$ & 84,532 & Yes, non-significant & 0.048 & 1.88 & 0.34 & -1.56 \\
\hline 149 & HMG1/2-like protein & $\begin{array}{l}\text { HMGL } \\
\text { WHE- } \\
\text { AT }\end{array}$ & 17,204 & No & 0.016 & 1.51 & 0.048 & 2.00 \\
\hline 11 & $\begin{array}{l}\text { Putative LRR disease-resistance protein/transmembrane } \\
\text { receptor kinase PS19 }\end{array}$ & $\begin{array}{l}\text { PS19 } \\
\text { PINST }\end{array}$ & 870 & No & 0.3 & 1.72 & 0.026 & 2 \\
\hline 13 & Endoplasmin & $\begin{array}{l}\text { ENPL } \\
\text { HORV- } \\
\text { U }\end{array}$ & 92,859 & Yes, significant & 0.57 & 2.27 & 0.043 & 2.44 \\
\hline 29 & Heat shock protein $81-1$ & $\begin{array}{l}\text { HSP81- } \\
\text { ORYSJ }\end{array}$ & 80,144 & Yes, significant & 0.39 & 2.20 & 0.049 & 5.96 \\
\hline 34 & Heat shock cognate $70 \mathrm{kDa}$ protein & $\begin{array}{l}\text { HSP7C- } \\
\text { PETH- } \\
\text { Y }\end{array}$ & 71,182 & No & 0.53 & 0.725 & 0.03 & 3.255 \\
\hline 41 & Putative F-box/FBD/LRR-repeat protein & $\begin{array}{l}\text { FDL48 } \\
\quad \text { ARAT- } \\
\text { H }\end{array}$ & 48,797 & Yes, significant & - & - & 0.019 & 1.99 \\
\hline 49 & $\begin{array}{l}\text { 4-hydroxy-7-methoxy-3-oxo-3,4-dihydro-2H-1,4-benzox } \\
\text { azin-2-yl glucoside beta-D-glucosidase 1a, chloroplast }\end{array}$ & $\begin{array}{c}\text { HGL1A_- } \\
\text { WHE- } \\
\text { AT }\end{array}$ & 64,467 & Yes, non-significant & 0.73 & -1.36 & 0.0019 & 1.90 \\
\hline 52 & Ribulose bisphosphate carboxylase (large chain) & $\begin{array}{l}\text { RBL } \\
\text { WHE- } \\
\text { AT }\end{array}$ & 52,817 & Yes, significant & 0.23 & -1.92 & 0.0056 & -1.85 \\
\hline 74 & UDP-glucose 6-dehydrogenase 4 & $\begin{array}{l}\text { UGDH4 } \\
\text { ORYSJ }\end{array}$ & 52,821 & Yes, non-significant & 0.2 & 1.43 & 0.00094 & 1.63 \\
\hline 77 & Probable ureidoglycolate hydrolase & $\begin{array}{l}\text { UAH_ } \\
\text { ORYSJ }\end{array}$ & 51,683 & Yes, non-significant & 0.026 & -1.59 & 0.046 & -2.02 \\
\hline 87 & Tricetin $3^{\prime}, 4^{\prime}, 5^{\prime}$-O-trimethyltransferase & $\begin{array}{l}\text { FOMT2- } \\
\text { WHE- } \\
\text { AT }\end{array}$ & 38,545 & Yes, non-significant & 0.9 & 0.18 & 0.042 & -1.85 \\
\hline 89 & Fructokinase-2 & $\begin{array}{l}\text { SCRK2 } \\
\text { ORYSJ }\end{array}$ & 35,494 & No & 0.95 & 0.04 & 0.018 & -2.08 \\
\hline 94 & Serine acetyltransferase 4 & $\begin{array}{l}\text { SAT4 } \\
\quad \text { ARAT- } \\
\text { H }\end{array}$ & 38,400 & Yes, significant & 0.21 & 1.695 & 0.013 & 1.605 \\
\hline 131 & Ras-related protein RABB1b & $\begin{array}{l}\text { RAB1B } \\
\text { ARAT- } \\
\text { H }\end{array}$ & 23,161 & Yes, non-significant & 0.07 & 1.64 & 0.00 & 1.54 \\
\hline
\end{tabular}

ChoInf, Choteau infested; ChoWT, Choteau wild type; $M W$, molecular weight; SchInf, Scholar infested; SchWT, Scholar wild type 


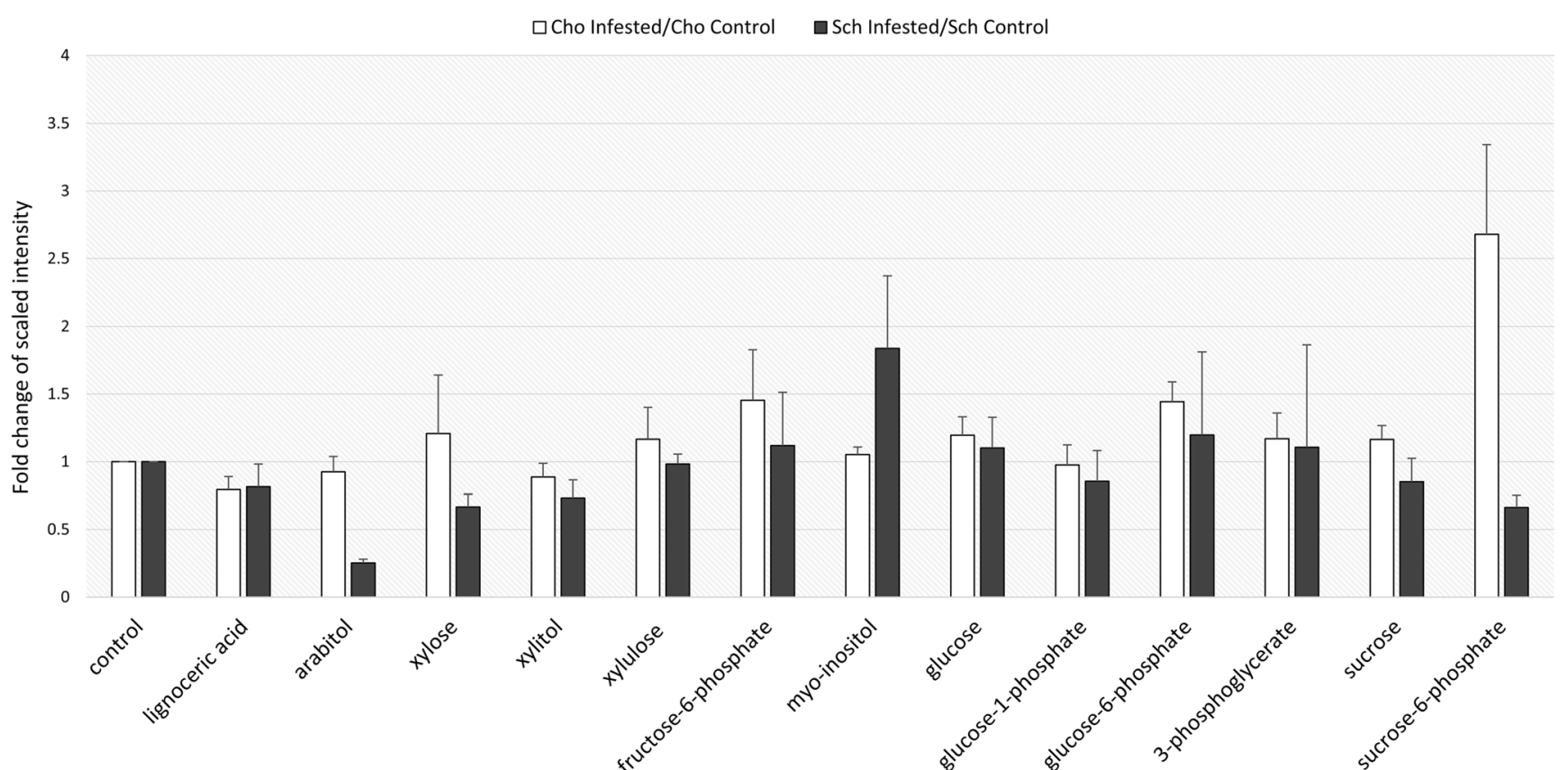

Fig. 5 Differentially regulated metabolites during WSS infestation. The differential regulation of metabolites belonging to the phenylpropanoid and pentose phosphate pathways, suggested as key pathways. The control for each metabolite is represented as 1 , while the fold changes for each

miRNA families, respectively, of which 14 were common to all three species (Fig. 3c). The miR1127 family from the Qss$3 B L$ locus in bread wheat targeted a single transcript from the same region, pointing to a potential auto-regulatory circuit (Supplementary Table S2a); however, this family is not well characterized in plants, and its target transcript does not exhibit homology to any known plant protein, so the functional aspects of such a circuit could not be determined. Similarly, three miRNA families from the durum wheat $Q s s-3 B L$ QTL, miR1135, miR1436, and miR1439, targeted three transcripts in the same region with no known homologs in the Viridiplantae (Supplementary Table S2b). In contrast to bread and durum wheat, none of the predicted miRNAs from the wild emmer wheat Qss-3BL QTL targeted transcripts within this region.

Both the miR1127 and miR1436 families also targeted transcripts outside the $Q s s-3 B L$ locus, including those involved in disease responses (Supplementary Table S2c). Small RNA interactions with putative disease-response genes were also found in the model grass species, Brachypodium (Lucas et al. 2014). Other putative miRNAs from the Qss$3 B L$ QTLs in the three species also targeted stress-responserelated transcripts encoded outside of this locus. Six miRNA families from the bread wheat $Q s s-3 B L$ QTL interacted with seven transcripts encoding disease-resistance proteins, while five miRNA families from the modern and wild tetraploid wheat $Q s s-3 B L$ loci each targeted seven transcripts within their transcriptomes, including disease-resistance-related transcripts. Notably, of all the miRNAs targeting disease-related transcripts, only the miR1117 and miR1439 families were metabolite were calculated by dividing the average scaled intensity value of the WSS-stressed samples by that of the WSS-control metabolite samples. Error bars were calculated by the basic rules of error propagation to estimate the average error in each sample

common to all three species. Although these observations imply a link between the $Q s s-3 B L$ QTL and miRNA-mediated regulation of certain stress response pathways, the functions and/or canonical target(s) of these miRNA families are largely unknown, complicating the elucidation of their regulatory pathways. The further characterization of wheat miRNAs and transcriptomes may unravel new dimensions to the WSS response that can be used in pest management and pesticide development.

\section{Differential regulation of QSS.msub-3BL-associated transcripts under WSS infestation}

DEGs located within these six physical regions were, in general, downregulated in response to WSS infestation, both in Choteau and Scholar, including genes potentially encoding proteins related to the stress response, cell signaling, protein turnover, and translation machineries, hinting at the metabolic events taking place in the plants. A total of 36 transcripts from the six physical regions defined by the QSS.msub-3BL molecular markers showed differential expression patterns in four pairwise comparison backgrounds: (1) Choteau-infested vs. control, (2) Scholar-infested vs. control, (3) Choteau-control vs. Scholarcontrol, and (4) Choteau-infested vs. Scholar-infested. The most dramatic change was observed in the infested vs. control Scholar stems, where 15 transcripts were differentially regulated. Since the semi-solid stems of the Scholar likely provide less resistance to larval growth, WSS infestation may be expected to trigger more dramatic changes in the metabolism of this cultivar; 
however, infestation appears to impede the biotic stress-related cellular processes. For instance, $C P R-5$ was upregulated in the Scholar stems upon infestation. CPR-5 is a plant response regulator that inhibits the production of PR proteins and salicylic acid, as well as the accumulation of reactive oxygen species, in the absence of biotic stress, reducing the cost of fitness (Rojas et al. 2014; Vos et al. 2013). Another transcript upregulated upon infestation encodes an aspartyl aminopeptidase, which has been linked to increased susceptibility to bacterial pathogens, as the silencing of this transcript resulted in delayed disease symptoms and decreased host cell death in Nicotiana benthamiana (Bae et al. 2013). On the other hand, another transcript encoding a different type of aminopeptidase, methionine aminopeptidase, was downregulated in infested Scholar stems. Although its role in the biotic stress response has not yet been reported, methionine aminopeptidase is an abiotic stress-related protein that can confer cold and freezing tolerance and aid in post-translational modifications (Jeong et al. 2011). Among the downregulated transcripts were two encoding LRR domain-containing proteins, one of which contained an NB-ARC domain as well. LRR-containing receptor kinases are well-known transmembrane proteins that recognize pathogen elicitors and activate the response pathways in the host plant (Huot et al. 2014). Similarly, the NB-ARC-LRRtype proteins are inducers of effector-triggered immunity in plants, as they sense the attack and turn on the response pathways (Takken and Goverse 2012). Moreover, two genes encoding enzymes responsible for the induction of vacuolar enzymes and defense-related molecules, including PR proteins and phytoalexins (Hatsugai et al. 2010), were also significantly downregulated in infested Scholar stems. While it is possible that, upon WSS infestation, Scholar stem cells may shut down mechanisms that would otherwise confer resistance against other stress factors to conserve energy, it could also be argued that the WSS eggs or larvae that managed to survive within the stem may manipulate the host defense systems in favor of their own survival. In addition to its already relatively less solid stem structure, the metabolism of Scholar appears to be highly vulnerable to WSS.

In the Choteau cultivar, a single transcript encoding a cysteine-rich receptor kinase was upregulated in response to WSS infestation, suggesting the activation of cell signaling pathways; however, similar to Scholar, the expression of a transcript encoding an LRR domain-containing protein kinase was repressed in the infested Choteau stems. Another strongly downregulated transcript upon WSS infestation encoded the FERONIA protein, which contains a malectin domain and belongs to a receptor kinase family. FERONIA is believed to be a sensor of cell wall integrity, playing a role in responding to cell wall disruption, such as wounding by herbivores (Savatin et al. 2014; Shih et al. 2014). WSS infestation therefore appears to suppress a number of stress-response pathway proteins in Choteau (Table 1, Supplementary Table S3), as was also observed in Scholar. Indeed, in several species, elicitors derived from insects or their symbiotic bacteria can inactivate or reduce the defense responses that would otherwise be triggered by herbivore chewing or egg deposition in the host plant, improving the herbivore's chance of survival (Bruessow et al. 2010; Chung et al. 2013; Hogenhout and Bos 2011). Nevertheless, the solid stems of Choteau still conferred a substantial level of tolerance against WSS in this cultivar.

Notably, under the control conditions, three of the four Qss$3 B L$ transcripts encoding potential stress-related proteins were more abundant in Choteau than Scholar. These transcripts encoded an LRR-domain-containing serine/threonine kinase, which is a transmembrane receptor with roles in sensing herbivore attack and activating the intracellular response pathways (Huot et al. 2014), a protein kinase with a thaumatin domain classified as a PR protein due to its activation in response to pathogen and insect attacks (Liu et al. 2010), and a $\mathrm{K}$ homology domain-containing protein. By contrast, one transcript, encoding a DUF1618 family protein, was less abundant in Choteau than Scholar. The DUF1618 family was recently found to be involved in the abiotic and biotic stress responses in rice, as several family members were repressed by salicylic acid, a key hormone that triggers plant stress responses (An and Mou 2011; Wang et al. 2014). The lower abundance of a DUF1618 family protein in Choteau stems under control conditions is therefore consistent with an elevated level of defense compared with Scholar, even in the absence of the pest. Taken together, the activity of defenserelated molecules in Choteau stems in comparison with Scholar may suggest a constitutively higher level of defense under normal growth conditions, stemming from the loci associated with WSS resistance. Upon infestation, the Choteau cultivar may benefit from the physical barrier provided by its solid stem, as well as its basal level of stress-response factors activated in advance, and may therefore be able to provide a quicker response and better resistance to oviposition or larval growth.

During WSS infestation, 10 DEGs were identified between the Choteau and Scholar cultivars. Infested Choteau stems contained more of a transcript encoding a 2,3bisphosphoglycerate-independent phosphoglycerate mutase than Scholar, which provides pyruvate to mitochondria in the glycolysis pathway to generate energy. Notably, a phosphoglycerate mutase was also upregulated in Arabidopsis thaliana in response to insect feeding (Zhang et al. 2010). A threonyl-tRNA synthetase-encoding transcript was almost sixfold more abundant in Choteau than Scholar, which may indicate highly active translation machinery in the cells of the infested Choteau stems. On the other hand, infested Choteau stems contained fewer transcripts encoding potential players in the biotic and abiotic stress tolerance of wheat, such as the receptor-like protein kinase FERONIA, the arabinosyltransferase ARAD1, the disease-resistance protein 
RGA3, and a rhodanese-like domain-containing protein. Like FERONIA, ARAD1 is associated with the cell wall; the cell walls of arad 1 mutants contain little arabinan, implying a role for ARAD1 in regulating the abundance of this important cell wall component (Sørensen et al. 2006). Rhodanase detoxifies reactive oxygen species that accumulate as a result of stress conditions, thereby protecting cellular compartments (Most and Papenbrock 2015). While the lower levels of transcripts encoding these proteins in infested Choteau stems compared with infested Scholar stems is intriguing, it is possible that solid stems of Choteau are less affected by the restricted larval growth and/or the Choteau stems may downregulate all nonspecific stress responses to conserve energy.

\section{Comprehensive proteomic and metabolomic analysis of the WSS response}

Our proteomics study suggested the differential regulation of 21 proteins, of which four were significantly differently produced following WSS infestation in both varieties (Table 2), suggesting an essential common role in the WSS response. HMG1/2-like protein was upregulated in both Choteau and Scholar. HMG proteins are associated with several important nuclear functions, such as DNA repair, recombination, and chromatin remodeling, through their interaction with histone proteins. Studies of Arabidopsis plants under abiotic stress indicated that epigenetic modifications arising from the activity of HMG proteins can play an important role in the response to stresses such as drought and cold (Cusanelli and Chartrand 2015; Kim et al. 2015; Štros et al. 2007). Both proteomic and transcriptomic data suggested the involvement of NBS-LRRassociated disease-resistance proteins in the WSS response, and the expression of such genes might be regulated by the activation of specific stress-response regions of the genome by the HMG proteins. Thus, manipulation of the HMG1/2-like protein might result in the activation of chromosomal sites specific to the insect response following WSS infestation, which would enable the plant to express certain genes involved in defense mechanisms.

Our proteomics analysis also revealed significant changes in the abundance of heat shock proteins (HSPs) and heat shock cognates (HSCs), important molecular chaperones responsible for protein folding, assembly, and degradation during many different cellular processes (Park and Seo 2015). In plants, they also contribute to the activity of the pathogen recognition receptors (PRRs) by aiding their accumulation for the activation of further stress responses (Nekrasov et al. 2009; Park and Seo 2015). HSP70/HSC70 was shown to play a particularly crucial role in the biotic stress response through its involvement in the hypersensitive response (HR; Kanzaki et al. 2003; Park and Seo 2015). The significant accumulation of HSP81-1/HSC70 under WSS stress in Scholar may facilitate the recognition of WSSspecific PRRs and the activation of the HR. On the other hand,
HSP81-1/HSC70 might aid protein folding and the elimination of misfolded proteins to protect cellular integrity under WSS stress. The increase in endoplasmin, a member of the HSP90 family that aids protein folding in the endoplasmic reticulum, also suggested changes in protein metabolism and the level of unfolded proteins. Endoplasmin production was also upregulated in response to powdery mildew infection, which further supports its involvement in the biotic stress response (Gupta and Tuteja 2011). Overall, these results suggest that the semisolid cultivar Scholar is highly affected at the molecular level by WSS larva infestation, which threatens its cellular homeostasis, whereas the solid-stemmed Choteau cultivar is more likely to resist the effects of the infestation.

At the proteomic level, several proteins possessing enzymatic function were differentially regulated, suggesting their further involvement in metabolomic pathways. One of these, a probable ureidoglycolate hydrolase, was significantly downregulated in both Choteau and Scholar under WSS stress. This enzyme functions in nitrogen metabolism by hydrolyzing ureidoglycolate into glyoxylate, carbon dioxide, and two other ammonia products ( $\mathrm{Li}$ et al. 2015). The regulation of ureide metabolism by the activity of ureidoglycolate hydrolase results in the accumulation of allantoin and allantoate, primary ureide products that accumulate in response to several abiotic stresses (Li et al. 2015; Watanabe et al. 2014). The reduced levels of ureidoglycolate hydrolase under WSS stress might be associated with the accumulation of primary ureide products such as allantoin, which could be used by the plant to generate a stronger signal to further enhance its metabolism for survival.

Furthermore, the proteomic and metabolomic data suggested changes in the phenylpropanoid pathway through the significant downregulation of PAL in solid-stemmed Choteau. The phenylpropanoid pathway is involved in the production of many important secondary metabolites, such as flavonoids and lignin. Lignin protects plants against mechanical damage in stress conditions, including drought or wounding (Vogt 2010), as it is an essential constituent of the secondary cell wall that reinforces vessel hydrophobicity and fibers and provides a physical barrier against pathogens (Vélez-Bermúdez et al. 2015). Under WSS stress, the regulation of the phenylpropanoid pathway is expected to occur in hexaploid wheat as a means of increasing physical support in the stems; however, the downregulation of the PAL enzyme suggests a reverse manipulation of this pathway. To gain a deeper understanding of lignin biosynthesis regulation under WSS stress, we examined the metabolic byproducts of the phenylpropanoid pathway in Scholar and Choteau. A slight decrease in lignoceric acid was detected in both varieties, which suggests that lignin content decreased coincidentally with the decrease in PAL detected at the proteomic level. The wheat stem lignin and cellulose contents and their effect on WSS lodging have already been analyzed in several papers; however, no significant correlation between these 
molecules and lodging resistance was identified in wheat (Kong et al. 2013). A study in maize (Zea mays) suggested a correlation between lignin content and brittle snap stalk breakage (Li 1997), which could indicate that hexaploid wheat might regulate its lignin content to decrease the risk of stalk breakage on the sides of stem thinned by larval feeding activity. Since the mechanical resistance of the stem tissue restricts larval development and feeding, the WSS larvae might generate chemicals that inhibit the production of PAL and secondary metabolites such as lignin, interfering with the plant stress response to increase their chances of survival. The significantly reduced levels of PAL in solid-stemmed Choteau particularly support this hypothesis, since this pith-filled variety restricts the space available for larval growth and reduces their survival; however, further experiments, particularly involving larval enzymes and metabolites, are required to fully comprehend the contribution of the phenylpropanoid secondary metabolite pathway to the WSS response.

Another significantly downregulated phenylpropanoidpathway enzyme in Scholar under WSS stress was tricetin 3',4',5'-O-trimethyltransferase, which catalyzes a methyl transfer reaction using the flavone tricetin $\left(5,7,3^{\prime}, 4^{\prime}, 5^{\prime}\right.$ pentahydroxyflavone) as a substrate to generate $3^{\prime}$ monomethyl-3',5'-dimethyl-(tricin), and 3',4',5'-trimethylether derivatives (Moheb et al. 2013). In rice, tricin appears to act as a natural biocide; the accumulation of tricin had an anti-feeding and anti-oviposition effect on a rice insect pest, the brown planthopper (Nilaparvata lugens; Bing et al. 2007), and it provided a fungicidal effect against several fungal diseases of this cereal, including rice seedling rot disease (Kong et al. 2004, 2010). The observed decrease in the amount of tricetin $3^{\prime}, 4^{\prime}, 5^{\prime}$-O-trimethyltransferase suggests a reduced level of tricin, which would further support the feeding of the larvae and oviposition in the Scholar plants. This enzyme has also been associated with lignin biosynthesis because of its ability to react with 5-hydroxyferulic acid. Moheb et al. (2013) found that tricetin $3^{\prime}, 4^{\prime}, 5^{\prime}$-O-trimethyltransferase accumulated despite the declining tricin levels in plants under cold stress, suggesting its secondary role in lignin biosynthesis that could support secondary cell wall production. Thus, the downregulation of tricetin $3^{\prime}, 4^{\prime}, 5^{\prime}$-O-trimethyltransferase will affect both tricin and lignin biosynthesis, promoting larval growth inside Scholar stems.

Secondary metabolite pathway associated with PAL was also found to be differentially regulated in Scholar upon WSS infestation; 4-hydroxy-7-methoxy-3-oxo-3,4-dihydro2H-1,4-benzoxazin-2-yl glucoside beta-D-glucosidase 1a (DIMBOA glucoside beta-D-glucosidase), which is responsible for the conversion of DIMBOA glucoside to DIMBOA and Dglucose (Sue et al. 2006), was significantly downregulated in Scholar stems. DIMBOA is a well-known toxic compound produced by many cereals as a defense against herbivores, including aphids, rootworms, and caterpillars (Martos et al. 1992;
Niemeyer 2009; Song et al. 2011). Feeding the European corn borer (Ostrinia nubilalis), an important pest of maize, with different concentrations of DIMBOA increased larval mortality and delayed pupation in a dose-dependent manner (Campos et al. 1989). In many other studies, DIMBOA was shown to be detrimental to aphids fed with artificial media containing this compound, and aphids preferred leaves with a lower DIMBOA content (Wouters et al. 2016). The downregulation of the DIMBOA glucoside beta-D-glucosidase enzyme suggests a decrease in the content of DIMBOA in the Scholar stems that would further promote larval feeding inside these tissues. This may arise from the interference of the WSS larvae with the WSS response in these plants, which was also suggested by the observation of their effects on lignin biosynthesis.

Another metabolomic pathway that may be differentially regulated under WSS stress was the pentose phosphate pathway, which supports both energy metabolism via glycolysis and the formation of structural sugars such as xylose and arabinose for further mechanical support. Glyceraldehyde-3phosphate dehydrogenase 1 , which is required for the conversion of glyceraldehyde-3-phosphate to 1,3bisphosphoglycerate during glycolysis, was significantly upregulated in both Choteau and Scholar, together with a slight increase in the amount of 3-phosphoglycerate, another metabolite produced during glycolysis. This supports the previous suggestion that WSS infestation increases the energy metabolism of the plant (Muñoz-Bertomeu et al. 2010). Additionally, a significant increase in glucose-6-phosphate and fructose-6-phosphate was observed in both cultivars, particularly Choteau; however, no significant change in the amount of glucose was detected. In Scholar, the level of UDP-glucose 6-dehydrogenase 4 was increased by WSS infestation, while the level of fructokinase-2 was halved, suggesting several regulatory effects on the glucuronic acid pathway during herbivore attack. In this pathway, glucose-6phosphate is first converted to glucose-1-phosphate, then UDP-glucose is formed and converted by UDP-glucose 6-dehydrogenase into UDP-glucuronic acid, a precursor of four structural sugars involved in cell wall biosynthesis, xylulose, xylitol, xylulose, and arabinose (Reboul et al. 2011). In accordance with the proteomic data, a decrease in the amount of xylose was detected in Scholar, while the xylitol and xylulose levels were slightly affected by WSS infection in both Scholar and Choteau. By contrast, a significant decrease was detected in the abundance of arabitol, the alcohol form of arabinose, in Scholar, but not in Choteau. Based on this observation, it is tempting to propose that the semi-solid stems of Scholar and the solid stems of Choteau have a different structural sugar metabolic response to WSS. The proteomic data indicate that Scholar may activate the UDP-glucose pathway to produce structural sugars to provide further mechanical support upon WSS infestation; however, the expected decrease in the amount of xylose and arabitol sugars was not 
observed, suggesting that these sugars are rapidly consumed for secondary cell wall formation. Another method of synthesizing UDP-glucose and supporting the gluconic acid pathway is to oxidize myo-inositol. Several studies have suggested that myo-inositol-derived UDP-glucose production might be specifically induced in plant cells under sugar starvation to maintain metabolic homeostasis (Valluru and Van den Ende 2011). In Scholar but not in Choteau, a significant increase in myoinositol abundance was observed following WSS infestation, suggesting that myo-inositol is involved in sugar metabolism in this cultivar. Since Choteau was already more resistant to WSS infestation because of its pith-filled stem structure, its WSS response does not appear to involve activation of the cell wall-associated mechanical tolerance pathways to the same extent as does Scholar. The upregulation of fructokinase-2 in Scholar stems further supports this observation, since this enzyme has important roles in vascular development and secondary cell wall formation (Stein et al. 2016). Instead of structural sugars, Choteau might instead activate sugar metabolism to produce more energy, which could explain its significant increase in sucrose-6-phosphate.

\section{Putative model of the WSS response in hexaploid wheat}

Our multiomics approach suggested a putative model for the WSS response in hexaploid wheat in which stem solidness is a key regulator (Fig. 6).
In the putative model of the WSS response, plant hormones (possibly cytokinin) activate a specific response through receptors located in the cell membrane. The activation of heat shock proteins and endoplasmin may also further support this recognition process. The signal is further relayed through the action of a variety of protein kinases, which specifically activate or deactivate mediator proteins through phosphorylation. These mediators then trigger the translocation of specific transcription factors into the nucleus to regulate gene expression. In addition to cellular signaling, chromosomal remodeling may also be involved in the activation of stress-responsive gene expression. This response results in changes to the metabolic events that occur in subcellular compartments, such as the peroxisomes, vacuoles, and chloroplasts. Based on our observations from the multiomics data, it is possible that WSS larvae may target this sequence of events to their benefit. Indeed, oviposition effectors are capable of modulating plant systems, including plant hormones, to suppress the host immune response (Erb et al. 2012). Several studies have provided evidence of herbivorous insects secreting proteins in their saliva that enter plant cells and inhibit the defense signaling pathways (Hogenhout and Bos 2011; Win et al. 2012). The restriction of larval growth by the solid stems of Choteau may result in them being less exposed to the larval effectors, or this cultivar may maintain an active basal level of signaling that can be augmented upon recognition of the larvae, providing better resistance against the developing WSS larvae.

In our model, two metabolic pathways are responsible for the response to WSS: the phenylpropanoid and pentose sugar

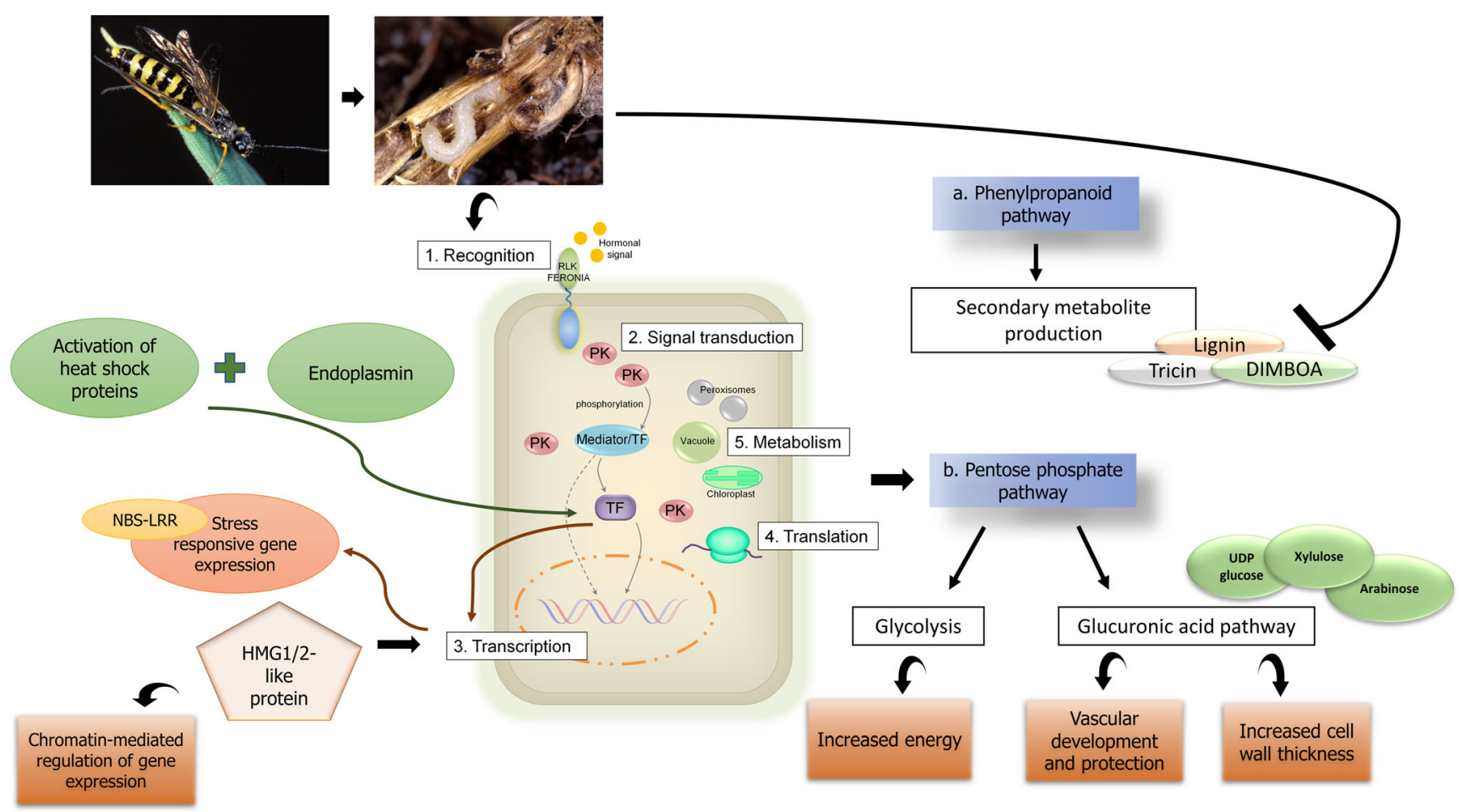

Fig. 6 A putative model for the WSS response in hexaploid wheat. PK, protein kinase; RLK, receptor-like kinase; TF, transcription factor 
pathways. The phenylpropanoid pathway produces secondary metabolites that support the mechanical defense against WSS, while also being involved in the production of antibiotic-like compounds that restrict larval growth. The pentose-sugar pathway produces both energy, to support survival under stress conditions, and structural sugar compounds, to provide further mechanical support. Differences in the stem solidness trait may be linked to changes in both of these pathways, since more mechanical support is needed by hollow and semi-solid varieties. Interference with the phenylpropanoid pathway may inhibit the production of compounds such as DIMBOA and tricin, which have an anti-feeding effect on larval growth and oviposition.

Thus, even though stem solidness is an important trait determining the mechanical tolerance to WSS in wheat, the plant's response to this pest is more complex than simple mechanical restriction of larval growth. We are currently analyzing the functions of some of the identified genes using TILLING and CRISPR mutants. Expanding our understanding of the WSS response mechanisms should further elucidate the interaction between wheat and WSS, facilitating the development of more effective pest control strategies.

Author contributions HB conceived the idea of the study and supervised all analyses. SB performed the RNA-Seq experiments and identified differentially expressed genes and miRNAs. BA analyzed the proteomic and metabolomic data. BAA conducted the comparative and syntenic analyses in wheat species. HB, SB, BA, and BAA drafted the manuscript. SB, $\mathrm{BA}, \mathrm{AV}$, and $\mathrm{MH}$ performed the WSS infestation and sample collections and edited the manuscript. DW and BB critically edited the manuscript.

Funding This study was funded by a Montana Wheat and Barley Committee Grant no. CY5417-462 and the Winifred-Asbjornson Plant Science Endowment. The Proteomics, Metabolomics, and Mass Spectrometry facility at Montana State University received support from the Murdock Charitable Trust and NIGMS of the National Institutes of Health (P20GM103474). The authors acknowledge Robert Peterson for the WSS images.

\section{Compliance with ethical standards}

Conflict of interest The authors declare that they have no conflict of interest.

Open Access This article is distributed under the terms of the Creative Commons Attribution 4.0 International License (http:// creativecommons.org/licenses/by/4.0/), which permits unrestricted use, distribution, and reproduction in any medium, provided you give appropriate credit to the original author(s) and the source, provide a link to the Creative Commons license, and indicate if changes were made.

\section{References}

Akpinar BA, Kantar M, Budak H (2015) Root precursors of microRNAs in wild emmer and modern wheats show major differences in response to drought stress. Funct. Integr. Genomics. 15(5):587598. https://doi.org/10.1007/s10142-015-0453-0

Alptekin B, Budak H (2017) Wheat miRNA ancestors: evident by transcriptome analysis of A, B, and D genome donors. Funct. Integr. Genomics 17:171-187. https://doi.org/10.1007/s10142-016-0487-y

Alptekin B, Akpinar A, Budak H (2016) A comprehensive prescription for plant miRNAs annotation. Front Plant Sci 7:2058. https://doi. org/10.3389/fpls.2016.02058

An C, Mou Z (2011) Salicylic acid and its function in plant immunity 53: 412-428. https://doi.org/10.1111/j.1744-7909.2011.01043.x

Anders S, Pyl PT, Huber W (2015) Genome analysis HTSeq - a python framework to work with high-throughput sequencing data. Bioinformatics 31(2):166-169. https://doi.org/10.1093/ bioinformatics/btu638

Ani Akpinar B, Yuce M, Lucas S, Vrána J, Burešová V, Doležel J, Budak H (2015) Molecular organization and comparative analysis of chromosome 5B of the wild wheat ancestor Triticum dicoccoides. Sci Rep 5(1):10763. https://doi.org/10.1038/srep10763

Avni R, Nave M, Barad O, Baruch K, Twardziok SO, Gundlach H, Hale I, Mascher M, Spannagl M, Wiebe K, Jordan KW, Golan G, Deek J, Ben-Zvi B, Ben-Zvi G, Himmelbach A, MacLachlan RP, Sharpe AG, Fritz A, Ben-David R, Budak H, Fahima T, Korol A, Faris JD, Hernandez A, Mikel MA, Levy AA, Steffenson B, Maccaferri M, Tuberosa R, Cattivelli L, Faccioli P, Ceriotti A, Kashkush K, Pourkheirandish M, Komatsuda T, Eilam T, Sela H, Sharon A, Ohad N, Chamovitz DA, Mayer KFX, Stein N, Ronen G, Peleg Z, Pozniak CJ, Akhunov ED, Distelfeld A (2017) Wild emmer genome architecture and diversity elucidate wheat evolution and domestication. Science 357(6346):93-97. https://doi.org/10.1126/science. aan0032

Bae C, Kim S, Lee DJ, Choi D (2013) Multiple classes of immune-related proteases associated with the cell death response in pepper plants. 8 . https://doi.org/10.1371/journal.pone.0063533

Beres BL, Dosdall LM, Weaver DK, Cárcamo HA, Spaner DM (2011a) Biology and integrated management of wheat stem sawfly and the need for continuing research. Can Entomol 143(2):105-125. https:// doi.org/10.4039/n10-056

Beres BL, Cárcamo HA, Yang RC, Spaner DM (2011b) Integrating spring wheat sowing density with variety selection to manage wheat stem sawfly. Agron J 103(6):1755-1764. https://doi.org/10.2134/ agronj2011.0187

Beres B, Cárcamo H, Byers J, Clarke F, Ruan Y, Pozniak C et al (2013) Host plant interactions between wheat germplasm source and wheat stem sawfly Cephus cinctus Norton (Hymenoptera: Cephidae). II. Other germplasm. Can J Plant Sci 93(6):1169-1177. https://doi.org/ 10.4141/cjps2013-035

Bing L, Hongxia D, Maoxin Z, Di X, Jingshu W (2007) Potential resistance of tricin in rice against brown planthopper Nilaparvata lugens (Stål). Acta Ecol Sin 27(4):1300-1306. https://doi.org/10.1016/ S1872-2032(07)60031-6

Bruessow F, Gouhier-darimont C, Buchala A, Metraux J, Reymond P (2010) Insect eggs suppress plant defence against chewing herbivores. 876-885. https://doi.org/10.1111/j.1365-313X.2010.04200.x

Budak H, Akpinar BA (2015) Plant miRNAs: biogenesis, organization and origins. Funct Integr Genomics 15(5):523-531. https://doi.org/ 10.1007/s10142-015-0451-2

Budak H, Akpinar BA, Unver T, Turktas M (2013) Proteome changes in wild and modern wheat leaves upon drought stress by twodimensional electrophoresis and nanoLC-ESI-MS/MS. Plant Mol Biol 83(1-2):89-103. https://doi.org/10.1007/s11103-013-0024-5

Budak H, Kantar M, Bulut R (2015) Stress responsive miRNAs and isomiRs in cereals. Plant Sci 235:1-13. https://doi.org/10.1016/j. plantsci.2015.02.008

Buteler M, Peterson RKD, Hofland ML, Weaver DK (2015) A multiple decrement life table reveals that host plant resistance and parasitism 
are major causes of mortality for the wheat stem sawfly. Environ Entomol 44(6):1571-1580. https://doi.org/10.1093/ee/nvv128

Cagirici HB, Biyiklioglu S, Budak H (2017a) Assembly and annotation of transcriptome provided evidence of miRNA mobility between wheat and wheat stem sawfly. Front Plant Sci 8:1653. https://doi. org/10.3389/fpls.2017.01653

Cagirici HB, Alptekin B, Budak H (2017b) RNA sequencing and coexpressed long non-coding RNA in modern and wild wheats. Sci Rep 7(1):10670. https://doi.org/10.1038/s41598-017-11170-8

Campos F, Atkinson J, Arnason JT, Philogène BJR, Morand P, Werstiuk $\mathrm{NH}$ et al (1989) Toxicokinetics of 2,4-dihydroxy-7-methoxy-1,4benzoxazin-3-one (DIMBOA) in the European corn borer, Ostrinia nubilalis (Hübner). J Chem Ecol 15(7):1989-2001. https://doi.org/ 10.1007/BF01207432

Choulet F, Alberti A, Theil S et al (2014) Structural and functional partitioning of bread wheat chromosome 3B. Science 345: 1249721. https://doi.org/10.1126/science.1249721

Chung SH, Rosa C, Scully ED, Peiffer M, Tooker JF, Hoover K, Luthe DS, Felton GW (2013) Herbivore exploits orally secreted bacteria to suppress plant defenses. Proc Natl Acad Sci 110(39):15728-15733. https://doi.org/10.1073/pnas.1308867110

Cusanelli E, Chartrand P (2015) Telomeric repeat-containing RNA TERRA: a noncoding RNA connecting telomere biology to genome integrity. Front Genet 6:1-9. https://doi.org/10.3389/fgene.2015. 00143

Dai X, Zhao PX (2011) PsRNATarget: a plant small RNA target analysis server. Nucleic Acids Res 39(suppl 2):W155-W159. https://doi. org/10.1093/nar/gkr319

Delaney KJ, Weaver DK, Peterson RKD (2010) Photosynthesis and yield reductions from wheat stem sawfly (Hymenoptera: Cephidae): interactions with wheat solidness, water stress, and phosphorus deficiency. J Econ Entomol 103(2):516-524. https://doi.org/10.1603/ Ec09229

Erb M, Meldau S, Howe GA (2012) Role of phytohormones in insectspecific plant reactions. Trends Plant Sci 17(5):250-259. https://doi. org/10.1016/j.tplants.2012.01.003

Esen A (1978) A simple method for quantitative, semiquantitative, and qualitative assay of protein. Anal. Biochem. 89:264-273. https://doi. org/10.1016/0003-2697(78)90749-2

Fiehn O, Wohlgemuth G, Scholz M, Kind T, Lee DY, Lu Y et al (2008) Quality control for plant metabolomics: Reporting MSI-compliant studies. Plant J. 53:691-704. https://doi.org/10.1111/j.1365-313X. 2007.03387.x

Gupta D, Tuteja N (2011) Chaperones and foldases in endoplasmic reticulum stress signaling in plants. Plant Signal Behav 6(2):232-236. https://doi.org/10.4161/psb.6.2.15490

Hatsugai N, Hara-nishimura I, Hatsugai N, Hara-nishimura I (2010) Two vacuole-mediated defense strategies in plants. 2324:2-5. https://doi. org $/ 10.4161 / p s b .5 .12 .13319$

Hogenhout SA, Bos JIB (2011) Effector proteins that modulate plantinsect interactions. Curr Opin Plant Biol 14(4):422-428. https:// doi.org/10.1016/j.pbi.2011.05.003

Huot B, Yao J, Montgomery BL, Yang S (2014) Growth-defense tradeoffs in plants: a balancing act to optimize fitness. Mol Plant 7(8):1267-1287. https://doi.org/10.1093/mp/ssu049

International Wheat Genome Sequencing Consortium (2014) A chromosome based draft sequencing of the hexaploid bread wheat (Triticum aestivum) genome. Science 345(6194): 1251788

Jeong H, Shin JS, Ok SH (2011) Plant science barley DNA-binding methionine aminopeptidase, which changes the localization from the nucleus to the cytoplasm by low temperature, is involved in freezing tolerance. Plant Sci 180(1):53-60. https://doi.org/10.1016/ j.plantsci.2010.09.004

Kantar M, Akpinar BA, Valárik M, Lucas SJ, Doležel J, Hernández P et al (2012) Subgenomic analysis of microRNAs in polyploid wheat.
Funct. Integr. Genomics 12(3):465-479. https://doi.org/10.1007/ s10142-012-0285-0

Kanzaki H, Saitoh H, Ito A, Fujisawa S, Kamoun S, Katou S, Yoshioka H, Terauchi R (2003) Cytosolic HSP90 and HSP70 are essential components of INF1-mediated hypersensitive response and nonhost resistance to pseudomonas cichorii in Nicotiana benthamiana. Mol Plant Pathol 4(5):383-391. https://doi.org/10.1046/j.13643703.2003.00186.x

Kim J-M, Sasaki T, Ueda M, Sako K, Seki M (2015) Chromatin changes in response to drought, salinity, heat, and cold stresses in plants. Front Plant Sci 6:114. https://doi.org/10.3389/fpls.2015.00114

Kong C, Xu X, Zhou B, Hu F, Zhang C, Zhang M (2004) Two compounds from allelopathic rice accession and their inhibitory activity on weeds and fungal pathogens. Phytochemistry 65(8):1123-1128. https://doi.org/10.1016/j.phytochem.2004.02.017

Kong CH, Xu XH, Zhang M, Zhang SZ (2010) Allelochemical tricin in rice hull and its aurone isomer against rice seedling rot disease. Pest Manag Sci 66(9):1018-1024. https://doi.org/10.1002/ps.1976

Kong E, Liu D, Guo X, Yang W, Sun J, Li X, Zhan K, Cui D, Lin J, Zhang A (2013) Anatomical and chemical characteristics associated with lodging resistance in wheat. Crop J 1(1):43-49. https://doi.org/10. 1016/j.cj.2013.07.012

Kozomara A, Griffiths-Jones S (2011) MiRBase: integrating microRNA annotation and deep-sequencing data. Nucleic Acids Res 39(Database):D152-D157. https://doi.org/10.1093/nar/gkq1027

Kurtoglu KY, Kantar M, Budak H (2014) New wheat microRNA using whole-genome sequence. Funct Integr Genomics 14(2):363-379. https://doi.org/10.1007/s10142-013-0357-9

Lesieur V, Martin JF, Weaver DK, Hoelmer KA, Smith DR, Morrill WL, Kadiri N, Peairs FB, Cockrell DM, Randolph TL, Waters DK, Bon MC (2016) Phylogeography of the wheat stem sawfly, Cephus cinctus Norton (Hymenoptera: Cephidae): implications for pest management. PLoS One 11(12):1-19. https://doi.org/10.1371/ journal.pone. 0168370

Li M (1997) Anatomical and morphological characteristics of maize genotypes varying in resistance to brittlesnap (unpublished master's thesis). University of Nebraska, Lincoln, USA

Li J, Qin R-Y, Li H, Xu R-F, Yang Y-C, Ni D-H, Ma H, Li L, Wei PC, Yang JB (2015) Low-temperature-induced expression of rice ureidoglycolate amidohydrolase is mediated by a C-repeat/dehydration-responsive element that specifically interacts with rice $\mathrm{C}$ repeat-binding factor 3. Front Plant Sci 6:1-11. https://doi.org/10. 3389/fpls.2015.01011

Liu J-J, Sturrock R, Ekramoddoullah AKM (2010) The superfamily of thaumatin-like proteins: its origin, evolution, and expression towards biological function. 419-436. https://doi.org/10.1007/ s00299-010-0826-8

Lucas SJ, Budak H (2012) Sorting the wheat from the chaff: identifying miRNAs in genomic survey sequences of Triticum aestivum chromosome 1AL. PLoS One 7(7):e40859. https://doi.org/10.1371/ journal.pone.0040859

Lucas S, Durmaz E, Akpnar BA, Budak H (2011) The drought response displayed by a DRE-binding protein from Triticum dicoccoides. Plant Physiol Biochem 49(3):346-351. https://doi.org/10.1016/j. plaphy.2011.01.016

Lucas SJ, Bestas K, Budak H (2014) Exploring the interaction between small RNAs and $\mathrm{R}$ genes during Brachypodium response to Fusarium culmorum infection. Gene 536(2):254-264. https://doi. org/10.1016/j.gene.2013.12.025

Martos A, Givovich A, Niemeyer HM (1992) Effect of DIMBOA, an aphid resistance factor in wheat, on the aphid predator Eriopis connexa Germar (Coleoptera: Coccinellidae). J Chem Ecol 18(3): 469-479. https://doi.org/10.1007/BF00994245

Moheb A, Agharbaoui Z, Kanapathy F, Ibrahim RK, Roy R, Sarhan F (2013) Tricin biosynthesis during growth of wheat under different 
abiotic stresses. Plant Sci 201-202:115-120. https://doi.org/10. 1016/j.plantsci.2012.12.005

Most P, Papenbrock J (2015) Possible roles of plant sulfurtransferases in detoxification of cyanide, reactive oxygen species, selected heavy metals and arsenate. 1410-1423. https://doi.org/10.3390/ molecules20011410

Muñoz-Bertomeu J, Cascales-Miñana B, Alaiz M, Segura J, Ros R (2010) A critical role of plastidial glycolytic glyceraldehyde-3phosphate dehydrogenase in the control of plant metabolism and development. Plant Signal Behav 5(1):67-69. https://doi.org/10. 1104/pp.109.143701.lycolysis

Nekrasov V, Li J, Batoux M, Roux M, Chu Z-H, Lacombe S, Rougon A, Bittel P, Kiss-Papp M, Chinchilla D, van Esse HP, Jorda L, Schwessinger B, Nicaise V, Thomma BPHJ, Molina A, Jones JDG, Zipfel C (2009) Control of the pattern-recognition receptor EFR by an ER protein complex in plant immunity. EMBO J 28(21):3428-3438. https://doi.org/10.1038/emboj.2009.262

Niemeyer H (2009) Hydroxamic acids derived from 2-hydroxy-2 H-1, 4benzoxazin-3 (4 H)-one: key defense chemicals of cereals. J Agric Food Chem 3(5):1677-1696. https://doi.org/10.1021/jf8034034

Nilsen KT, Clarke JM, Beres BL, Pozniak CJ (2016) Sowing density and cultivar effects on pith expression in solid-stemmed durum wheat. Agron J 108(1):219-228. https://doi.org/10.2134/agronj2015.0298

Nilsen KT, Diaye AN, Maclachlan PR, Clarke JM, Ruan Y, Cuthbert RD, et al. (2017) High density mapping and haplotype analysis of the major stem-solidness locus SSt1 in durum and common wheat. 1-19

Park C-J, Seo Y-S (2015) Heat shock proteins: a review of the molecular chaperones for plant immunity. Plant Pathol J 31(4):323-333. https://doi.org/10.5423/PPJ.RW.08.2015.0150

Portman SL, Krishnankutty SM, Reddy GVP (2016) Entomopathogenic nematodes combined with adjuvants presents a new potential biological control method for managing the wheat stem sawfly, Cephus cinctus (Hymenoptera: Cephidae). PLoS One 11(12):e0169022. https://doi.org/10.1371/JOURNAL.PONE.0169022

Reboul R, Geserick C, Pabst M, Frey B, Wittmann D, Lütz-Meindl U, Léonard R, Tenhaken R (2011) Down-regulation of UDPglucuronic acid biosynthesis leads to swollen plant cell walls and severe developmental defects associated with changes in pectic polysaccharides. J Biol Chem 286(46):39982-39992. https://doi. org/10.1074/jbc.M111.255695

Rojas CM, Senthil-kumar M, Tzin V, Mysore KS (2014) Regulation of primary plant metabolism during plant-pathogen interactions and its contribution to plant defense. 5:1-12. https://doi.org/10.3389/fpls. 2014.00017

Savatin DV, Gramegna G, Modesti V, Cervone F (2014) Wounding in the plant tissue: the defense of a dangerous passage. Front Plant Sci 5:111. https://doi.org/10.3389/fpls.2014.00470

Shih H, Miller ND, Dai C, Spalding EP, Monshausen GB (2014) Report the receptor-like kinase FERONIA is required for mechanical signal transduction in Arabidopsis seedlings. Curr Biol 24(16):1887-1892. https://doi.org/10.1016/j.cub.2014.06.064

Song YY, Cao M, Xie LJ, Liang XT, Zeng RS, Su YJ, Huang JH, Wang RL, Luo SM (2011) Induction of DIMBOA accumulation and systemic defense responses as a mechanism of enhanced resistance of mycorrhizal corn (Zea mays L.) to sheath blight. Mycorrhiza 21(8): 721-731. https://doi.org/10.1007/s00572-011-0380-4

Sørensen SO, Orfila C, Pauly M, Harholt J, Kru J (2006) ARABINAN DEFICIENT 1 is a putative arabinosyltransferase involved in biosynthesis of pectic arabinan in Arabidopsis 1 [ W ]. Plant Physiol 140:49-58. https://doi.org/10.1104/pp.105.072744.1

Stein O, Avin-Wittenberg T, Krahnert I, Zemach H, Bogol V, Daron O, Aloni R, Fernie AR, Granot D (2016) Arabidopsis Fructokinases are important for seed oil accumulation and vascular development. Front Plant Sci 7:2047. https://doi.org/10.3389/fpls.2016.02047
Štros M, Launholt D, Grasser KD (2007) The HMG-box: a versatile protein domain occurring in a wide variety of DNA-binding proteins. Cell Mol Life Sci 64(19-20):2590-2606. https://doi.org/10. 1007/s00018-007-7162-3

Sue M, Yamazaki K, Yajima S, Nomura T, Matsukawa T, Iwamura H, Miyamoto T (2006) Molecular and structural characterization of hexameric b-D-glucosidases in wheat and rye. Plant Physiol 141(4):1237-1247. https://doi.org/10.1104/pp.106.077693

Takken FLW, Goverse A (2012) How to build a pathogen detector: structural basis of NB-LRR function. Curr Opin Plant Biol 15(4):375384. https://doi.org/10.1016/j.pbi.2012.05.001

van den Berg RA, Hoefsloot HCJ, Westerhuis JA, Smilde AK, van der Werf MJ (2006) Centering, scaling, and transformations: improving the biological information content of metabolomics data. BMC Genomics 7:142. https://doi.org/10.1186/1471-2164-7-142

Valluru R, Van den Ende W (2011) Myo-inositol and beyond - emerging networks under stress. Plant Sci 181(4):387-400. https://doi.org/10. 1016/j.plantsci.2011.07.009

Varella AC, Weaver DK, Sherman JD, Blake NK, Heo HY, Kalous JR, Chao S, Hofland ML, Martin JM, Kephart KD, Talbert LE (2015) Association analysis of stem solidness and wheat stem sawfly resistance in a panel of north American spring wheat germplasm. Crop Sci 55(5):2046-2055. https://doi.org/10.2135/cropsci2014.12.0852

Varella AC, Weaver DK, Peterson RKD, Sherman JD, Hofland ML, Blake NK, Martin JM, Talbert LE (2017) Host plant quantitative trait loci affect specific behavioral sequences in oviposition by a stem-mining insect. Theor Appl Genet 130(1):187-197. https:// doi.org/10.1007/s00122-016-2805-0

Vélez-Bermúdez I-C, Salazar-Henao JE, Fornalé S, López-Vidriero I, Franco-Zorrilla J-M, Grotewold E, Gray J, Solano R, Schmidt W, Pagés M, Riera M, Caparros-Ruiz D (2015) A MYB/ZML complex regulates wound-induced lignin genes in maize. Plant Cell 27(11): 3245-3259. https://doi.org/10.1105/tpc. 15.00545

Vogt T (2010) Phenylpropanoid biosynthesis. Mol Plant 3(1):2-20. https://doi.org/10.1093/mp/ssp106

Vos IA, Pieterse CMJ, Van Wees SCM (2013) Costs and benefits of hormone-regulated plant defences. Plant Pathol 62:43-55. https:// doi.org/10.1111/ppa.12105

Wang W, Vignani R, Scali M, Cresti M (2006) A universal and rapid protocol for protein extraction from recalcitrant plant tissues for proteomic analysis. Electrophoresis 27:2782-2786. https://doi.org/ 10.1002/elps.200500722

Wang L, Shen R, Chen LT, Liu YG (2014) Characterization of a novel DUF1618 gene family in rice. J Integr Plant Biol 56(2):151-158. https://doi.org/10.1111/jipb.12130

Watanabe S, Matsumoto M, Hakomori Y, Takagi H, Shimada H, Sakamoto A (2014) The purine metabolite allantoin enhances abiotic stress tolerance through synergistic activation of abscisic acid metabolism. Plant Cell Environ 37(4):1022-1036. https://doi.org/ $10.1111 /$ pce. 12218

Win J, Chaparro-Garcia A, Belhaj K, Saunders DGO, Yoshida K, Dong S et al (2012) Effector biology of plant-associated organisms: concepts and perspectives. Cold Spring Harb Symp Quant Biol 77, 235-247. https://doi.org/10.1101/sqb.2012.77.015933

Wouters FC, Blanchette B, Gershenzon J, Vassão DG (2016) Plant defense and herbivore counter-defense: benzoxazinoids and insect herbivores. Phytochem Rev 15(6):1127-1151. https://doi.org/10.1007/ s11101-016-9481-1

Wu TD, Watanabe CK (2005) GMAP: a genomic mapping and alignment program for mRNA and EST sequences. Bioinformatics 21(9): 1859-1875. https://doi.org/10.1093/bioinformatics/bti310

Zhang J, Sun L, Liu L, Lian J (2010) Proteomic analysis of interactions between the generalist herbivore Spodoptera exigua ( Lepidoptera: Noctuidae ) and Arabidopsis thaliana. 324-333. https://doi.org/10. 1007/s11105-009-0156-6 